\title{
Modeling and Parametric Analysis of a Unimorph Piezo- composite Energy Harvester with Interdigitated Electrodes
}

\author{
Ahmed Jemai $^{a}$, Fehmi Najar $^{a 1}$, Moez Chafra ${ }^{a, b}$, Zoubeida Ounaies $^{c}$ \\ a Applied Mechanics and Systems Research Laboratory, Tunisia Polytechnic School, \\ University of Carthage, B.P. 743, La Marsa 2078, Tunisia \\ ${ }^{b}$ Institut Préparatoire aux Etudes d'Ingénieurs d'El Manar, Université de Tunis El \\ Manar, 1002, Tunis, Tunisia. \\ ${ }^{c}$ Department of Mechanical and Nuclear Engineering, The Pennsylvania State Uni- \\ versity, 157B Hammond Building, University Park, PA 16802, USA.
}

\begin{abstract}
This study investigates the derivation of an accurate parameterized analytical model of a vibration-based energy harvester using piezocomposite material and interdigitated electrode. The derived model is used to analyze and optimize the harvested electrical energy under different resistance loads and excitation frequencies. The energy harvester is composed of a unimorph design cantilever beam partially covered by a piezocomposite material with interdigitated electrodes. The model provides an improved approach to optimize the performance of the system by taking into account the nonlinear electrical potential distribution and nonuniform vibration mode shapes over the beam's length due to the presence of the piezocomposite patch. We use a Galerkin procedure along with the Gauss's law to derive the analytical reduced-order model and study the dynamic response of the energy harvesting system. We demonstrate that different parameters are involved into the optimization process of the system such as the number of electrodes, the different layer thicknesses, the piezocomposite patch length, the fiber volume fraction and the substrate material. The proposed analysis shows that significant increase of the harvested energy could be obtained if all design parameters are correctly chosen. A numerical finite element model is also developed to validate the obtained analytical results.
\end{abstract}

\section{Keywords}

Energy harvesting, Unimorph, Piezocomposite, AFC, MFC, Interdigitated electrodes.

\section{Introduction}

Recent advances in the electronics industry have made wireless electronic devices require less power to operate. Thus, autonomous wireless systems can be developed if the

${ }^{1}$ Corresponding author, e-mail: fehmi.najar@ept.rnu.tn (F. Najar). 
required electrical energy to feed the system could be harvested from the environment. Many methods have been investigated to develop suitable power supply by converting an ambient energy source into electricity. Electric energy can be produced from light using photocells, from strain using magneto-electric or piezoelectric generators and from temperature gradient using thermo-couples. The abundance of vibrations in ambient environment, in addition to simplicity of integration and self-power capability of the piezoelectric transduction mechanism, encourage to give significant attention to piezoelectric vibration-based energy harvester (PVEH).

During the last decade, several researchers provided many efforts to increase the output electrical power of PVEH. Different approaches have been proposed to reach this goal ranging from proposing new mechanical designs to engineer new composite material structures. Next, we present some of these approaches.

In general, PVEH are composed of a mechanical bender in which the active piezoelectric material has been incorporated. An additional inertial component can be added to increase the transduced energy since it is proportional to the strain generated inside the bender through displacement due to the PVEH's vibration. This later case was studied by Jiang et al. [1]. They used a cantilever beam bender with a proof mass attached to its free end and studied the effect of physical and geometrical parameters of the system on the performance of the harvested electrical power. The PVEH is supposed to vibrate around its lowest natural frequency where the majority of environmental excitations frequencies are (from 20 to $200 \mathrm{~Hz}$ [2]). As a result, the maximum strain is generally confined near the clamped region of the bender. Therefore, researchers proposed to optimize the strain distribution along the bender's length in order to increase the harvested energy. Within this scope, Roundy et al. [3] demonstrated that a trapezoidal cantilever bender generate more than twice the electrical power compared to a classical rectangular shape. Ben Ayed et al. [4] investigated the effects of linear and quadratic shape variations of the PVEH. They concluded that, for specific electrical resistance loads, the quadratic shape can yield up to two times the energy harvested by a rectangular shape PVEH.

All the above mentioned research works can only harvest energy near the fundamental natural frequency of the bender. To increase the bandwidth at which the PVEH is efficient, Abdelkefi et al. [5] proposed a multimodal design of a bender that undergoes coupled bending-torsion vibrations. They showed that the proposed design yields an increase about $30 \%$ of the harvested power when compared to the case of an energy harvester undergoing bending only. Abdelkefi et al. [6] also demonstrated that their proposed design can be used for a multifrequency excitation without compromising the harvested electrical energy.

Other, looked at the arrangement of the piezoelectric layers inside the bender in order to optimize the strain distribution in the thickness direction. In this framework, Zheng and $\mathrm{Xu}[7]$ investigated an asymmetric air-spaced layers cantilever beam and they claimed that their proposed design increases the harvested electrical energy. Erturk et al. [8] studied analytically and experimentally a bimorph cantilever PVEH using different layers with independent electrodes. They concluded that the type of electrical connection (series or parallel) highly influence the harvested electrical energy. Erturk et al. [9] investigated the case of a unimorph PVEH with segmented 
electrodes in the longitudinal direction. They proved that an increase of the harvester performance is possible.

Interdigitated electrodes (IDE) could also be used to segment active layers and form juxtaposed regions of piezoelectric patches. This approach allows the use of the $d_{33}$ mode instead of the classical $d_{31}$ mode. It was shown that the $d_{33}$ mode could produce higher harvested power as shown in [10,11, 12]. Wihin this framework, Bent and Hagood [13] designed, in 1995, a new piezoelectric actuator where IDE have been integrated. They combine it with a piezoelectric composite material to increase the flexibility of the actuator, the new design is known as the Active-Fiber Composite (AFC). The proposed AFC piezocomposite is a combination of circular PZT fibers inclusion into an epoxy matrix. A Similar design was proposed by Wilkie et al [14] in 2000, but using square piezoelectric fiber instead of the circular cross section. Their piezoelectric actuator is known as Micro-Fiber Composite (MFC) [15].

Unfortunately, AFC and MFC based PVEH have weak performances. Sodano et al. [16] demonstrated experimentally that the electrical energy generated from a PVEH integrating IDE was very low when compared to a classical monolithic PZT PVEH with parallel electrodes. They justified this drawback by the reduced effective capacitance due to the IDE configuration. Sodano et al. $[17,18]$ presented experimental results showing that the current generated from PVEH integrating IDE was not sufficient to recharge small batteries. Therefore, it is necessary to identify the influential geometrical and physical parameters that contribute to the reduction of the harvested energy.

Besides the complexity of modeling and simulation of such energy harvester, because of the presence of the piezocomposite and the IDE configuration that produce complex electric field distribution, several attempts were made to propose analytical and numerical models of AFC and MFC. Bowen et al. [10] presented Finite Element (FE) analysis and optimization of AFC actuator assuming that the polarization is uniform and unidirectional. Beckert et al. [20] also developed an FE analysis, however they use an inhomogeneous poling state via a two-steps processing technique. It consists to examine first the electric field distribution which determines the local poling state in the piezocomposite, and second to update the electric field distribution according to the new polarization. However, these FE-based models are not suitable to optimize AFC and MFC based PVEH, especially when one wants to integrate the dynamic effects.

Mo et al. [21, 22] developed an analytical model of an IDE harvester with monolithic piezoelectric material assuming unidirectional and uniform electric field using a quasi-static approach. They presented a detailed parametric study to optimize the electrical charge and power generated by the harvester. Knight et al. [23] presented an optimization study of the same design implemented using a MEMS technology. They used the analytical model developed by Mo et al. [21] and introduced a poling correction factor to investigate the losses associated with non-uniform poling. They presented a parametric study in terms of electrode patterns, piezoelectric layer and electrode dimensions to examine their effects on the poling correction factor. Erturk et al. [24] investigated the use of MFC for energy harvesting. They presented a parametric study to investigate the effects of substrate material and piezoelectric layer 
thickness on the harvested power. They derived a semi-analytical model of the unimorph MFC harvester and introduced different correction factors in order to handle the non-uniform field into the MFC configuration. The developed model successfully predicted the dynamics of the MFC unimorph design especially for low values of electrical resistance load. Jemai et al. [25] developed an analytical and numerical model for a unimorph AFC harvester. They assumed unidirectional and uniform electric field between successive electrodes in the case of relatively large electrode separation and small electrode's width. They demonstrated that the proposed closed-form solution is in good agreement with FEM simulations. A similar analysis was developed, for the same PVEH in [26], when large displacements are considered.

Nevertheless, in most of the above mentioned studies where analytical solutions based on the Galerkin procedure are proposed, the presence of the piezoelectric patch is not taken into account to extract the mode shapes of the PVEH, either for the IDE configuration [24, 25] or for the classical parallel electrode configuration [27, 28, 29]. In fact, often the calculated mode shapes incorrectly assume that the substrate beam is fully covered by the piezoelectric patch.

In this study, an improvement of the analytical model found in [25] is proposed along with an extensive parametric analysis of the system's geometric and physical properties that influence the harvested electrical energy. First, the exact mode shapes are considered by taking into account the non-uniform beam thickness. Second, the proposed model accounts for non-uniform electric field between two consecutive IDE. Indeed, classical models of IDE harvesters consider only a uniform electric field that not only violates the Maxwell's equations but are also in conflict with numerical simulations done by FEM $[21,22,25]$. The derived analytical model and the obtained closed-form solutions are used to investigate the effects of various parameters on the harvested electrical power. The analytical results are validated by a numerical FEM solution developed using the commercial package ANSYS.

\section{Problem formulation of the PVEH with base excitation}

\subsection{Unimorph cantilever beam with AFC patch: Modeling strategy}

The considered PVEH is a unimorph cantilever beam in which the substrate layer is made of aluminum and the upper layer is an AFC piezocomposite patch partially covering the length of the beam near the clamped part (Figure 1).

The piezocomposite under study is formed by a transversely isotropic material (PZT-5A) embedded as circular fibers into an isotropic epoxy matrix (Figure 2). The material properties of the piezocomposite constituents are given in Table 1 , where $C_{i j}$ are the coefficients of elastic stiffness at constant electric field, $e_{i j}$ are the piezoelectric constants at constant strain and $\epsilon_{i j}$ are the electric permittivity constants at constant strain. The clamped end of the beam is subject to a transverse harmonic displacement $Y(t)=Y_{0} \sin (\Omega t)$ where $\Omega$ is the driving frequency and $Y_{0}$ is the amplitude (next we let $Y_{0}=0.1 \mathrm{~mm}$ ). The multilayer structure of the PVEH is shown in Figure 3 where each layer is defined by its position with respect to the neutral axis. These positions are determined by assuming that the total amount of the normal stress across the 


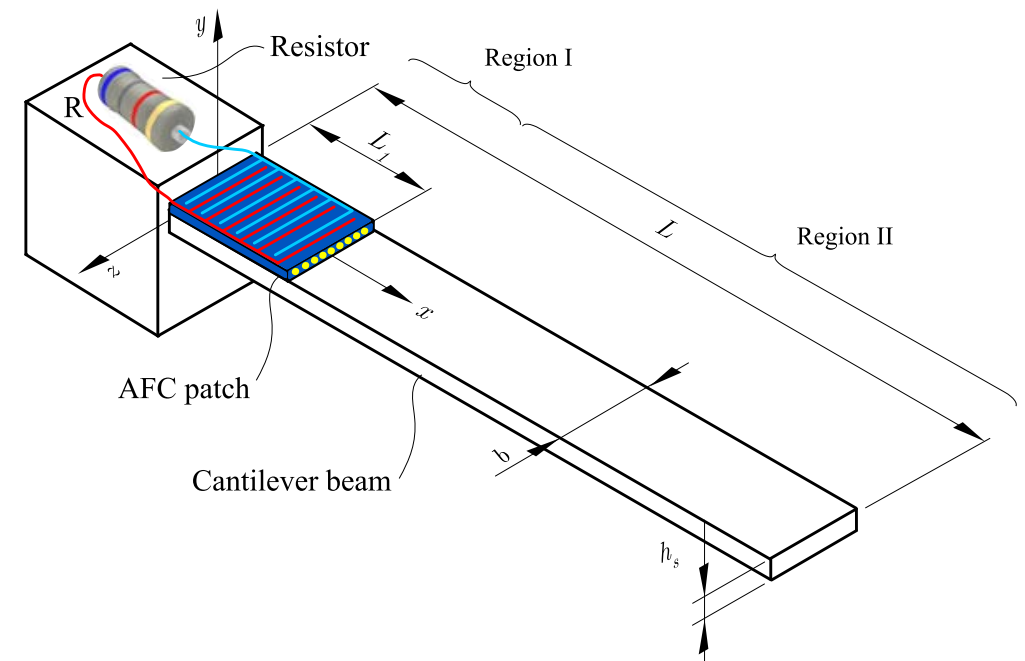

Figure 1: Schematic of the unimorph energy harvester bender using IDE.

Table 1: Material properties of the piezocomposite constituents: Fiber (PZT-5A) and Matrix (Epoxy)

\begin{tabular}{|c|c|c|c|c|c|}
\hline & $\begin{array}{c}C_{11} \\
(\mathrm{GPa})\end{array}$ & $\begin{array}{c}C_{12} \\
(\mathrm{GPa})\end{array}$ & $\begin{array}{c}C_{13} \\
(\mathrm{GPa})\end{array}$ & $\begin{array}{c}C_{33} \\
(\mathrm{GPa})\end{array}$ & $\begin{array}{c}C_{44} \\
(\mathrm{GPa})\end{array}$ \\
\hline PZT-5A & 120.35 & 75.18 & 75.1 & 110.87 & 21.05 \\
Epoxy & 9.84 & 7.75 & 7.75 & 9.84 & 0.86 \\
\hline \hline$C_{66}$ & $e_{31}$ & $e_{33}$ & $e_{15}$ & $\epsilon_{11}$ & $\epsilon_{33}$ \\
$(\mathrm{GPa})$ & $\left(\mathrm{C} / \mathrm{m}^{2}\right)$ & $\left(\mathrm{C} / \mathrm{m}^{2}\right)$ & $\left(\mathrm{C} / \mathrm{m}^{2}\right)$ & $\left(10^{-9} \mathrm{~F} / \mathrm{m}\right)$ & $\left(10^{-9} \mathrm{~F} / \mathrm{m}\right)$ \\
\hline 22.57 & -5.35 & 15.78 & 12.3 & 9.16 & 8.30 \\
0.86 & - & - & - & 0.885 & 0.885 \\
\hline
\end{tabular}

cross section is zero and neglect the effect of the voltage across the electrodes. These positions are given by [25] as follows:

$$
y_{0}=-\bar{y}, \quad y_{1}=h\left(1-\lambda_{h}\right)-\bar{y}, \quad y_{2}=h-\bar{y}
$$

where $\lambda_{h}=\frac{h_{p}}{h}$ is the thickness ratio and $\bar{y}$ is the neutral axis position given by

$$
\bar{y}=\frac{h}{2}\left(\left(1-\lambda_{h}\right)+\frac{\lambda_{h} \bar{C}_{33}^{e}}{\lambda_{h} \bar{C}_{33}^{e}+\left(1-\lambda_{h}\right) E_{s}}\right)
$$

where $h=h_{p}+h_{s}$ is the total thickness of the beam. $\bar{C}_{33}^{e}=C_{33}^{e}-\frac{C_{13}^{e}{ }^{2}}{C_{11}^{e}}$ and $E_{s}$ are respectively the effective Young's modulus of piezoelectric and substrate layers.

\subsection{Homogenization of the piezocomposite material in the AFC patch}

To derive the analytical model of the PVEH, we need to replace the piezocomposite material of the AFC patch by a homogenized equivalent material having the same 


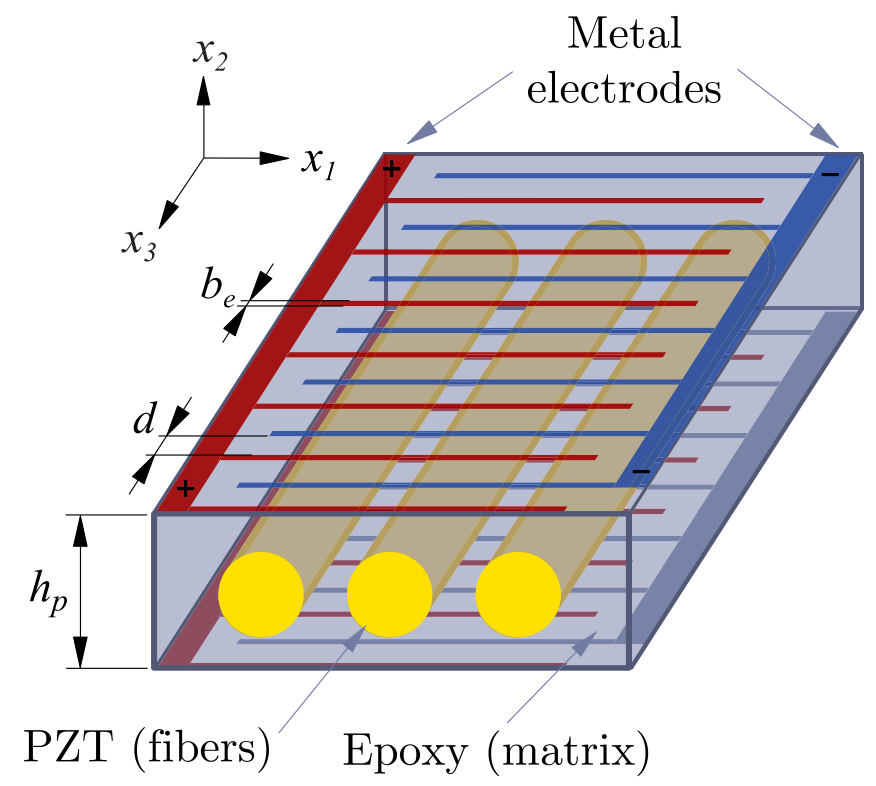

Figure 2: Schematic of the Active Fiber Composite.

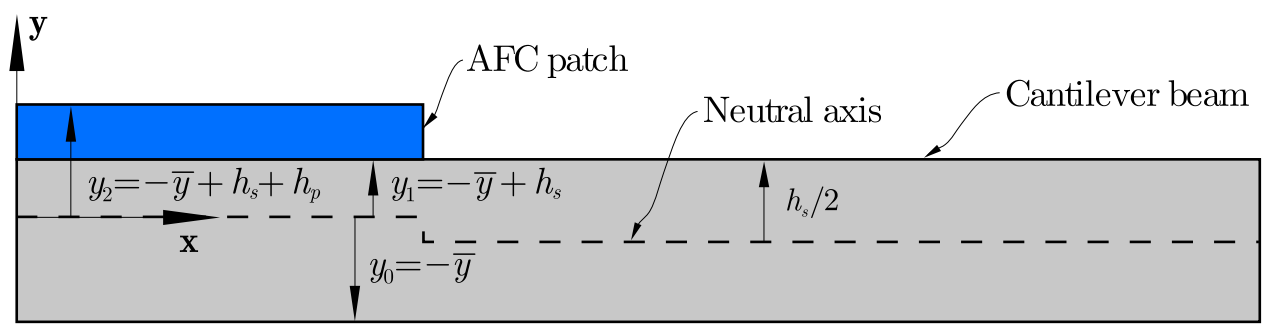

Figure 3: Neutral axis position

effective parameters than the original one. To estimate the new elastic constants, a periodic homogenization technique based on FEM is applied on a Representative Volume Element (RVE), shown in Figure 4-a. The 3D FEM model is composed of 22900 8-node coupled-field solid elements (SOLID5) with 24843 nodes. The periodic homogenization technique is based on a set of tensile and pure shear numerical tests applied to the RVE where a particular loading case return only one value in the strain or electric field tensors is non-zero and all others become zero, for more details see [30]. The same procedure can be applied to a MFC configuration to generate the equivalent homogenized piezoelectric material. Example of tensile test to extract the $C_{11}^{e}$ coefficient is shown in Figure 4-b.

The effective nonzero coefficients of the electromechanical constants matrix of the homogenized material, when assuming 50\% of fiber volume fraction (FVF) in the piezocomposite, are calculated using different test loads. The obtained results are shown in Table 2, where the superscript $e$ indicates effective values. In fact, the chosen FVF is obtained by examining a commercial AFC (from Advanced Cerametrics Inc). The FVF is experimentally determined based on scanning electron microscopy 
Table 2: Effective material properties of the homogenized material with 50\% FVF

\begin{tabular}{|c|c|c|c|}
\hline$C_{11}^{e}(\mathrm{GPa})$ & $C_{12}^{e}(\mathrm{GPa})$ & $C_{13}^{e}(\mathrm{GPa})$ & $C_{33}^{e}(\mathrm{GPa})$ \\
19.97 & 12.77 & 13.59 & 39 \\
\hline$C_{44}^{e}(\mathrm{GPa})$ & $C_{66}^{e}(\mathrm{GPa})$ & $e_{31}^{e}\left(\mathrm{C} / \mathrm{m}^{2}\right)$ & $e_{33}^{e}\left(\mathrm{C} / \mathrm{m}^{2}\right)$ \\
2.44 & 1.85 & -0.465 & 9.485 \\
\hline$e_{15}^{e}\left(\mathrm{C} / \mathrm{m}^{2}\right)$ & $\epsilon_{11}^{e}\left(10^{-9} \mathrm{~F} / \mathrm{m}\right)$ & $\epsilon_{33}^{e}\left(10^{-9} \mathrm{~F} / \mathrm{m}\right)$ & \\
0.43 & 2.33 & 4.69 & \\
\hline
\end{tabular}
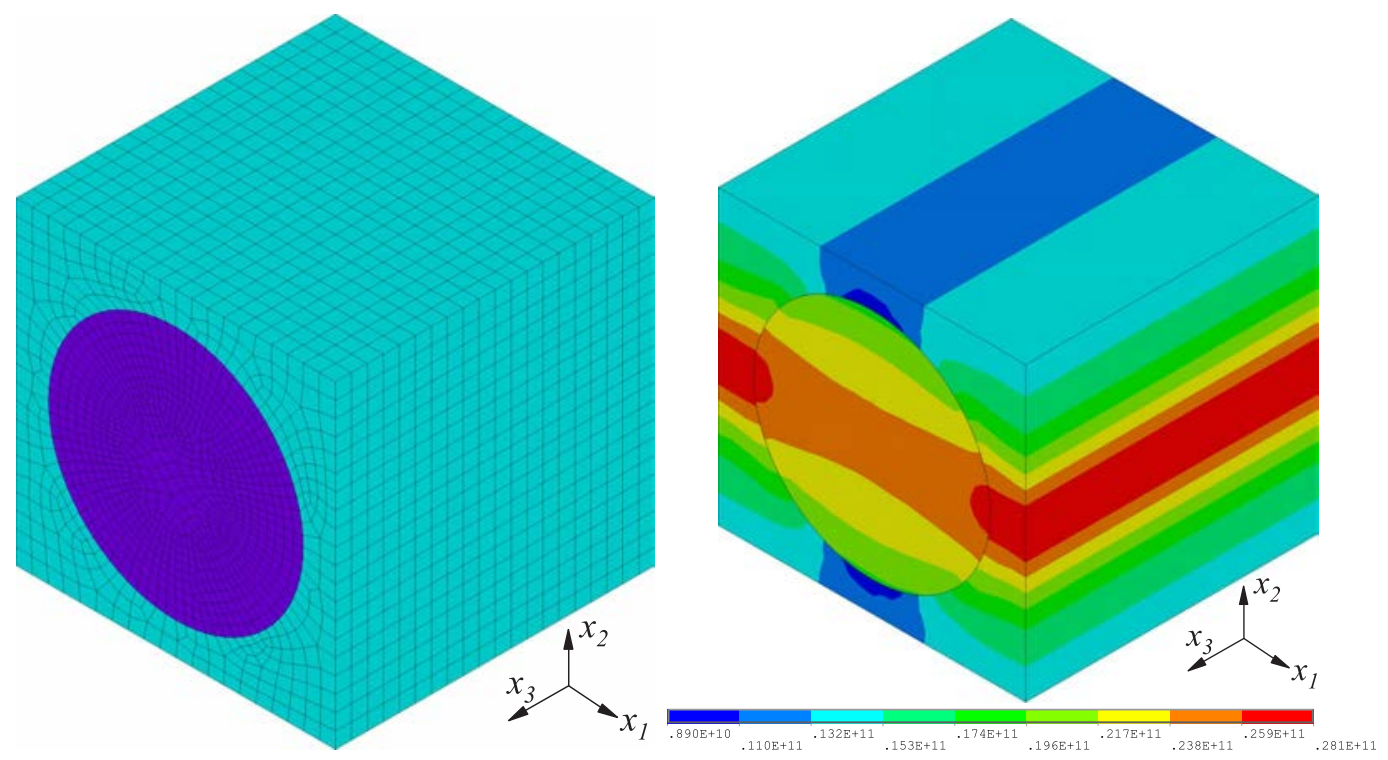

(a) 3D FE Model of the RVE with 50\% FVF. (b) Stress distribution in a tensile test in the $x_{1}$ direction

Figure 4: Representative Volume Element.

(SEM) images and assuming continuous fibers (such as the ratio of area contents was equivalent to the ratio of volume contents). The measured FVF values vary from $42 \%$ to $53 \%$ (Figure 5), the value of $50 \%$ is selected in this study. To obtain the micrographs shown in Figure 5, the AFCs were first freeze-fractured in liquid nitrogen, then imaged using a Hitachi SEM.

\subsection{Equation of motion}

To derive the equation of motion of the considered unimorph cantilever beam coupled to the equation that describes the electrical behavior of the AFC patch, with its IDE configuration along with the external electrical resistance load, one have to start by correctly represent the electrical field within two consecutive IDE.

Previous studies demonstrated that the electric field corresponding to IDE configuration is inhomogeneous and anisotropic. However, for relatively large electrode distance $d$ and small electrode's width $b_{e}$, the electric field could be considered unidi- 


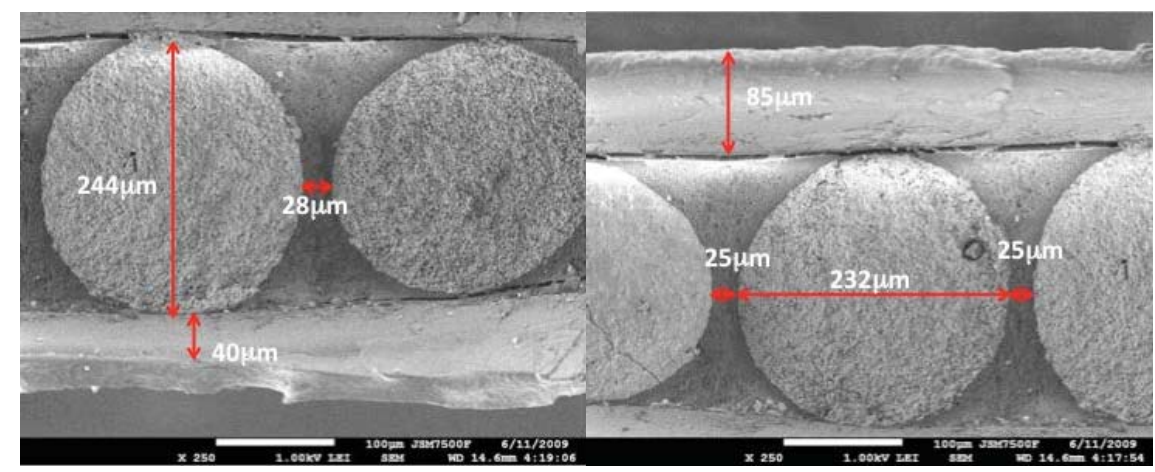

Figure 5: SEM images of the AFC.

rectional $[8,25]$. For the analytical analysis at hand, the surface IDE will be replaced by a set of transverse IDE in the $\left(x_{1}-x_{2}\right)$ plane (Figure 6 ). Consequently, the obtained electric field is unidirectional along the $x_{3}$-axis between two consecutive transverse IDE because of high aspect ratio.

Using a classical uniform field hypothesis between two electrodes [8], an analytical model was derived in [25] assuming linear voltage drop between two consecutive transverse IDE. However, such hypothesis violate Maxwell's equations for the electric displacement. To overcome this, an electric field that takes into account the self-field effect is proposed in this work. The new assumption will satisfy Maxwell's equations and is consistent with FE simulation of the electrical voltage drop between two consecutive electrodes (see the section 3 for more details).

The proposed unimorph design with IDE activates the $d_{33}$ mode in bending displacement of the beam. Therefore, assuming transversely isotropic material with a polarization along the $x$-axis (corresponding to the $x_{3}$-axis in the RVE local frame) and an unidirectional electric field in the same direction, the following constitutive equations are considered:

$$
\begin{aligned}
& \sigma_{11}^{p}=\bar{C}_{33}^{e} \varepsilon_{11}-\bar{e}_{33}^{e} E_{1} \\
& D_{1}=\bar{e}_{33}^{e} \varepsilon_{11}+\bar{\epsilon}_{33}^{e} E_{1}
\end{aligned}
$$

where $\sigma_{11}^{p}$ and $\varepsilon_{11}=-y v^{\prime \prime}(x, t)$ are, respectively, the normal stress and strain in the homogenized piezocomposite, $v(x, t)$ is the transverse displacement of the neutral axis of the beam at position $x$ and prime superscript denote derivation with respect to $x$. $\bar{e}_{33}^{e}=e_{33}^{e}-\frac{C_{13}^{e}}{C_{11}^{e}} e_{31}^{e}$ and $\bar{\epsilon}_{33}^{e}=\epsilon_{33}^{e}+\frac{e_{31}^{e}}{C_{11}^{e}}$ are, respectively, the piezoelectric constant at constant strain and the electric permittivity constant at constant strain of the homogenized piezocomposite, with plane-stress assumption.

The electric field $E_{1}(x, y)$ is taken along the $x$-axis. In fact, because of the IDE configuration, $E_{1}$ reverse its polarity each distance $d$. Therefore, the following expression of the electric field is proposed:

$$
E_{1}(x, y)=E_{1}^{*}(x, y) g(x)\left(H\left(y-y_{2}\right)-H\left(y-y_{1}\right)\right)
$$

where $H$ is the Heaviside step function, $g(x)$ is a function that accounts for the spatial distribution of the transverse IDE in the $x$ direction and $E_{1}{ }^{*}(x, y)$ is the local electric 

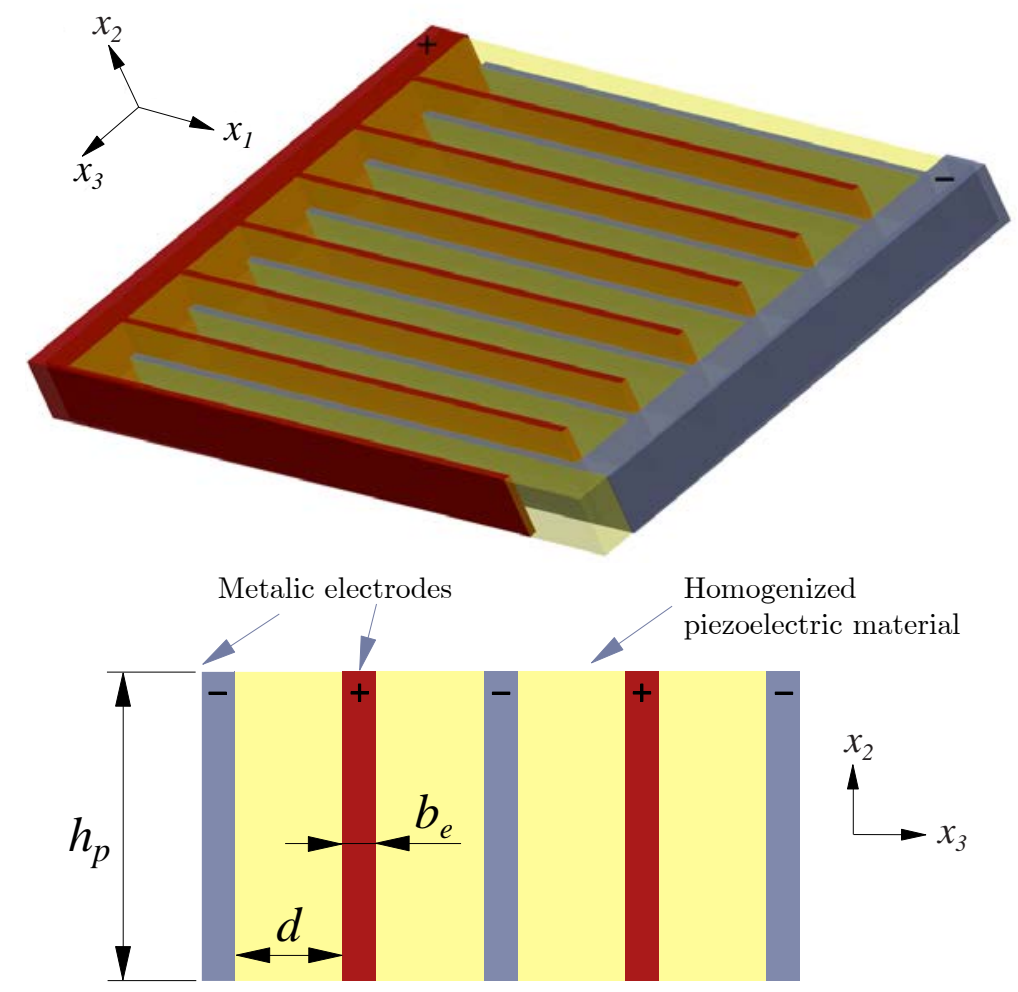

Figure 6: Homogenized AFC patch.

field given between the $i^{\text {th }}$ and the $i^{\text {th }}+1$ electrodes, they are given by

$$
\begin{gathered}
g(x)=\sum_{i=1}^{\frac{N-1}{2}}\{-2 H(x-(2 i-1) d)+H(x-2 i d)+H(x-(2 i-2) d)\} \\
E_{1}^{*}(x, y)=-\frac{V(t)}{d}-\frac{\bar{e}_{33}^{e}}{\bar{\epsilon}_{33}^{e}}\left(\varepsilon_{11}-\frac{1}{d} \int_{i d}^{(i+1) d} \varepsilon_{11} d x\right), i=0, \ldots, N-2
\end{gathered}
$$

where $N$ is the number of transverse IDE of both signs and $V(t)$ is the harvested voltage between two consecutive IDEs. It is worth noting here that $E_{1}{ }^{*}(x, y)$ is obtained first by assuming a constant field component in the $y$ direction due to the voltage between the electrodes, then by solving Equation (4) for the self-field component of the electric field.

The AFC patch along with substrate layer are modeled using the Euler-Bernoulli beam theory. The considered cantilever beam (Figure 1 ) have a total length $L$ while the AFC patch's length is $L_{1}=\delta_{h} L$ with $\delta_{h}$ is the length ratio. The beam is subdivided into two regions: Region I of length $L_{1}$, where unimorph configuration is adopted, and region II of length $L-L_{1}$ where only the substrate layer is present. 


\subsubsection{Mechanical modeling}

Using the Hamilton's principle applied to the proposed structure and following the same steps as in [25], the equation of motion and associated boundary conditions of the unimorph cantilever beam including the AFC patch are obtained as follows:

$$
\begin{gathered}
\left(H_{I} m_{I}+H_{I I} m_{I I}\right)(\ddot{v}+\ddot{Y})+c \dot{v}+\left(K_{y} v^{\prime \prime}+b \frac{\bar{e}_{33}^{e}}{3 \bar{\epsilon}_{33}^{e}}\left(y_{2}^{3}-y_{1}^{3}\right) g v^{\prime \prime}\right)^{\prime \prime} H_{I} \\
+\left(E_{s} \frac{b}{3}\left(y_{1}^{3}-y_{0}^{3}\right) v^{\prime \prime \prime \prime}\right) H_{I I}-b \bar{e}_{33} \frac{V(t)}{2 d} g^{\prime \prime}\left(y_{2}^{2}-y_{1}^{2}\right) H_{I} \\
-b \frac{\bar{e}_{33}^{e}}{3 \bar{\epsilon}_{33}^{e}}\left(y_{2}{ }^{3}-y_{1}{ }^{3}\right) \frac{g^{\prime \prime}}{d(N-1)}\left(\sum_{i=0}^{N-2} \int_{i d}^{(i+1) d} v^{\prime \prime} d x\right) H_{I}=0 \\
v(0, t)=0, v^{\prime}(0, t)=0, v^{\prime \prime}(L, t)=0, \text { and } v^{\prime \prime \prime}(L, t)=0
\end{gathered}
$$

where prime denote derivative with respect to $x$ and overdot derivative with respect to $t, H_{I}=H(x)-H\left(x-L_{1}\right), H_{I I}=H\left(x-L_{1}\right)-H(x-L), m_{I}=\rho_{s} b h_{s}+\rho_{p} b h_{p}$, $m_{I I}=\rho_{s} b h_{s}$ are the mass per unit length for regions (I and II) respectively, $c$ is the damping coefficient and $K_{y}$ is the bending stiffness for region I, it is given by

$$
K_{y}=K_{y s}+K_{y p}=\frac{b E_{s}}{3}\left(y_{1}^{3}-y_{0}^{3}\right)+\frac{b \bar{C}_{33}^{e}}{3}\left(y_{2}^{3}-y_{1}^{3}\right)
$$

where $K_{y s}$ is the stiffness of substrate layer in region I and $K_{y p}$ is the stiffness of the AFC patch alone.

To solve for the transverse displacement $v(x, t)$ and the harvested voltage $V(t)$, we use the Galerkin procedure that yields a reduced-order model describing the dynamic behavior of the system near the excited modes. We start by solving the free undamped eigenvalue problem at SC conditions, without taking into account the self-field effect. The associated eigenvalue problem is given by letting $v(x, t)=\phi(x) e^{i \omega t}$, that is

$$
-\omega^{2}\left(H_{I} m_{I}+H_{I I} m_{I I}\right) \phi(x)+\left[K_{y} H_{I}+E_{s} \frac{b}{3}\left(y_{1}^{3}-y_{0}^{3}\right) H_{I I}\right] \phi^{\prime \prime \prime \prime}(x)=0
$$

Next, we start the application of the Galerkin procedure by expressing the transverse displacement $v(x, t)$ as follows:

$$
v(x, t)=\sum_{r=1}^{\infty} \phi_{r}(x) \eta_{r}(t)
$$

where $\eta_{r}(t)$ is the $r^{t h}$ modal mechanical response and $\phi_{r}(x)$ is the $r^{t h}$ mode shape of the eigenvalue problem given by Equation (10). This equation should be solved for region I and II, thus, the mode shapes are split into two functions given by

$$
\begin{aligned}
\phi_{r}(x) & =\left\{\begin{array}{l}
\phi_{r}^{I}(x), \text { for } 0 \leq x \leq L_{1} \\
\phi_{r}^{I I}(x), \text { for } L_{1} \leq x \leq L
\end{array}\right. \\
& =\left\{\begin{array}{l}
A_{r}^{I} \sin \left(\beta_{r}^{I} x\right)+B_{r}^{I} \cos \left(\beta_{r}^{I} x\right)+C_{r}^{I} \sinh \left(\beta_{r}^{I} x\right)+D_{r}^{I} \cosh \left(\beta_{r}^{I} x\right) \\
A_{r}^{I I} \sin \left(\beta_{r}^{I I} x\right)+B_{r}^{I I} \cos \left(\beta_{r}^{I I} x\right)+C_{r}^{I I} \sinh \left(\beta_{r}^{I I} x\right)+D_{r}^{I I} \cosh \left(\beta_{r}^{I I} x\right)
\end{array}\right.
\end{aligned}
$$


where $A_{r}^{I}, B_{r}^{I}, C_{r}^{I}, D_{r}^{I}, A_{r}^{I I}, B_{r}^{I I}, C_{r}^{I I}$ and $D_{r}^{I I}$ are constants to be determined for each region,

$$
\beta_{r}^{I}=\left(\omega_{r}^{2} \frac{m_{I}}{K_{y}}\right)^{\frac{1}{4}} \quad \text { and } \quad \beta_{r}^{I I}=\left(\omega_{r}^{2} \frac{m_{I I}}{E_{s} \frac{b}{3}\left(y_{1}^{3}-y_{0}^{3}\right)}\right)^{\frac{1}{4}}
$$

are the $r^{\text {th }}$ frequency numbers for regions I and II. They are calculated by using the following boundary conditions obtained from Equation (9):

$$
\begin{aligned}
& \phi_{r}^{I}(0)=0 \text { and } \phi_{r}^{I^{\prime}}(0)=0 \\
& \phi_{r}^{I I^{\prime \prime}}(L)=0 \text { and } \phi_{r}^{I I^{\prime \prime \prime}}(L)=0
\end{aligned}
$$

along with the continuity conditions between regions I and II,

$$
\begin{aligned}
& \phi_{r}^{I}\left(L_{1}\right)=\phi_{r}^{I I}\left(L_{1}\right) \\
& \phi_{r}^{I^{\prime}}\left(L_{1}\right)=\phi_{r}^{I I^{\prime}}\left(L_{1}\right) \\
& K_{y} \phi_{r}^{I^{\prime \prime}}\left(L_{1}\right)=E_{s} \frac{b}{3}\left(y_{1}^{3}-y_{0}^{3}\right) \phi_{r}^{I I^{\prime \prime}}\left(L_{1}\right) \\
& K_{y} \phi_{r}^{I^{\prime \prime \prime}}\left(L_{1}\right)=E_{s} \frac{b}{3}\left(y_{1}^{3}-y_{0}^{3}\right) \phi_{r}^{I I^{\prime \prime \prime}}\left(L_{1}\right)
\end{aligned}
$$

Substituting Equation (12) into the Equation of motion (8), multiplying by the mode shape, integrating over the beam's length and using the orthogonality conditions, we end up with a set of ODE describing the modal response of the beam, they are given by

$$
M_{r} \ddot{\eta}_{r}(t)+C_{r} \dot{\eta}_{r}(t)+K_{r} \eta_{r}(t)+F_{r}=0
$$

In Equation 20, the modal mass is given by

$$
M_{r}=m_{I} \int_{0}^{L_{1}} \phi_{r}^{I}(x)^{2} d x+m_{I I} \int_{L_{1}}^{L} \phi_{r}^{I I}(x)^{2} d x
$$

The modal mechanical stiffness is $K_{r}=K_{r}^{I}+K_{r}^{I I}+K_{r}^{e}$ where $K_{r}^{I}$ and $K_{r}^{I I}$ are the modal mechanical stiffness terms for region I and II, expressed as

$$
\begin{aligned}
& K_{r}^{I}=K_{r}^{I s}+K_{r}^{I p}=K_{y s} \int_{0}^{L_{1}} \phi_{r}^{I^{\prime \prime \prime \prime \prime}}(x) \phi_{r}^{I}(x) d x+K_{y p} \int_{0}^{L_{1}} \phi_{r}^{I^{\prime \prime \prime \prime \prime}}(x) \phi_{r}^{I}(x) d x(22) \\
& K_{r}^{I I}=E_{s} \frac{b}{3}\left(y_{1}^{3}-y_{0}^{3}\right) \int_{L_{1}}^{L} \phi_{r}^{I I^{\prime \prime \prime \prime \prime}}(x) \phi_{r}^{I I}(x) d x
\end{aligned}
$$

and $K_{r}^{e}$ is the modal electromechanical stiffness induced by the electric self-field; it is given by

$$
\begin{aligned}
& K_{r}^{e}=d \chi \int_{0}^{L_{1}}\left(g(x) \phi_{r}^{I^{\prime \prime}}(x)\right)^{\prime \prime} \phi_{r}^{I}(x) d x \\
& -\frac{\chi}{(N-1)} \int_{0}^{L_{1}}\left(g(x) \sum_{i=0}^{N-2} \int_{d i}^{d(i+1)} \phi_{r}^{I^{\prime \prime}}(x) d x\right)^{\prime \prime} \phi_{r}^{I}(x) d x
\end{aligned}
$$


where $\chi=b \frac{\bar{e}_{33}^{e}{ }^{2}}{3 \bar{\epsilon}_{33}^{e} d}\left(y_{2}^{3}-y_{1}^{3}\right)$ is the higher-order coupling coefficient.

The modal damping is given by

$$
C_{r}=C_{r}^{I}+C_{r}^{I I}=\int_{0}^{L_{1}} c^{I} \phi_{r}^{I}(x)^{2} d x+\int_{L_{1}}^{L} c^{I I} \phi_{r}^{I I}(x)^{2} d x
$$

In order to fulfill compatibility of the proposed damping model with the one implemented in ANSYS, when the proposed model will be validated in section 3, we calculate the modal damping by assuming Rayleigh damping with stiffness proportionality to material, that is

$$
C_{r}^{I}=\beta^{p} K_{r}^{I p}+\beta^{s} K_{r}^{I s}
$$

and

$$
C_{r}^{I I}=\beta^{s} K_{r}^{I I}
$$

Here, $\beta^{p}$ and $\beta^{s}$ are the material-dependent damping multipliers for the AFC patch and the substrate layer materials, respectively.The modal damping ratio is obtained by the following relation

$$
\zeta_{r}=\beta^{p} \frac{K_{r}^{I p}}{2 \omega_{r} M_{r}}+\beta^{s} \frac{K_{r}^{I s}+K_{r}^{I I}}{2 \omega_{r} M_{r}}
$$

The modal force $F_{r}$, in Equation 20, is expressed as

$$
F_{r}=m_{r} \ddot{Y}(t)+f_{r} V(t)
$$

where

$$
\begin{aligned}
& m_{r}=m_{I} \int_{0}^{L_{1}} \phi_{r}^{I}(x) d x+m_{I I} \int_{L_{1}}^{L} \phi_{r}^{I I}(x) d x \\
& f_{r}=\kappa \int_{0}^{L_{1}} g^{\prime \prime}(x) \phi_{r}^{I}(x) d x
\end{aligned}
$$

and $\kappa=-b \frac{\bar{e}_{33}^{e}}{2 d}\left(y_{2}^{2}-y_{1}^{2}\right)$ is the coupling coefficient.

\subsubsection{Electrical modeling}

The IDE configuration is adopted by connecting in parallel the transverse electrodes (Figure 6). Therefore, one can electrically model the AFC patch as a set of capacitors and current sources connected in parallel, where the harvested voltage $V(t)$ is measured across the external load resistance $R$ (Figure 7).

The Gauss's law is used here with the above mentioned assumptions concerning the electric field distribution. As a result, the harvested voltage is directly related to the bending displacement of the cantilever beam. The Kirchhoff law is applied for the equivalent electrical circuit given in Figure 7 and the Galerkin approximation is used to obtained the discretized equation describing the electrical behavior [25]. It is given by

$$
C_{P} \dot{V}(t)+\frac{V(t)}{R}=I(t)
$$




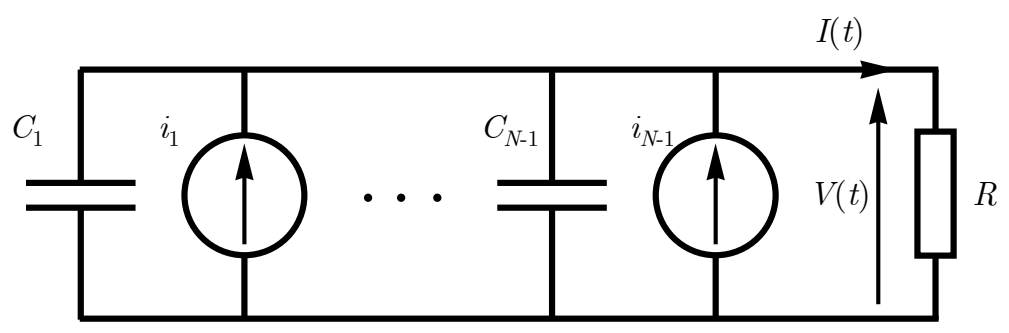

Figure 7: Electrical model of the AFC

where the internal equivalent capacitance term $C_{P}$ is expressed as

$$
C_{P}=(N-1)^{2} \bar{\epsilon}_{33}^{e} \frac{b h_{p}}{L_{1}}
$$

and the equivalent current source $I(t)$ is given by

$$
I(t)=\sum_{r=1}^{\infty} \kappa_{r} \dot{\eta}_{r}(t) \quad \text { where } \quad \kappa_{r}=\left.\kappa \sum_{i=1}^{N-1} \frac{d \phi_{r}^{I}(x)}{d x}\right|_{x=(i-1) d} ^{x=i d}
$$

\subsubsection{Closed-form solutions of the PVEH}

Assuming harmonic base motions around the fundamental mode shape at frequency $\Omega$, the steady state modal mechanical response of the beam and the steady state voltage response across the resistive load are also assumed to be harmonic at the same frequency. As a result, for a sinus type excitation only the imaginary part of the solution is retained, the voltage and modal displacement are respectively given by

$$
V(t)=\frac{\sum_{r=1}^{\infty} \frac{\kappa_{r} m_{r} \Omega^{2} Y_{0}}{j \Omega C_{r}-M_{r} \Omega^{2}+K_{r}}}{C_{p}-\frac{j}{\Omega R}+\sum_{r=1}^{\infty} \frac{\kappa_{r} f_{r}}{j \Omega C_{r}-M_{r} \Omega^{2}+K_{r}}} e^{j \Omega t}
$$

and

$$
v_{k}(x, t)=\sum_{r=1}^{\infty} \frac{\phi_{r}(x) \Omega^{2} Y_{0}}{j \Omega C_{r}-M_{r} \Omega^{2}+K_{r}}\left(m_{r}-\frac{f_{r} \sum_{r=1}^{\infty} \frac{\kappa_{r} m_{r}}{j \Omega C_{r}-M_{r} \Omega^{2}+K_{r}}}{C_{p}-\frac{j}{\Omega R}+\sum_{r=1}^{\infty} \frac{\kappa_{r} f_{r}}{j \Omega C_{r}-M_{r} \Omega^{2}+K_{r}}}\right) e^{j \Omega t}
$$

where $j=\sqrt{-1}$, and $\phi_{r}(x)=\phi_{r}^{I}(x)$ for region I and $\phi_{r}(x)=\phi_{r}^{I I}(x)$ for region II.

\section{Model validation and comparison with classical approaches}

The closed-form solution obtained for the mechanical and electrical responses given by Equations (30) and (31), provide an interesting tool to study the PVEH performance for different design configurations. The material and geometrical properties of the PVEH are displayed in Table 3. 
Table 3: Material and geometrical properties of the bi-layered cantilever beam

\begin{tabular}{|c|c|c|c|c|}
\hline $\begin{array}{c}L \\
(\mathrm{~mm})\end{array}$ & $\begin{array}{c}L_{1} \\
(\mathrm{~mm})\end{array}$ & $\begin{array}{c}b \\
(\mathrm{~mm})\end{array}$ & $\begin{array}{c}h_{p} \\
(\mathrm{~mm})\end{array}$ & $\begin{array}{c}h_{s} \\
(\mathrm{~mm})\end{array}$ \\
\hline 100 & 60 & 20 & 0.33 & 1 \\
\hline $\begin{array}{c}\rho_{p} \\
\left(\mathrm{~kg} / \mathrm{m}^{3}\right)\end{array}$ & $\begin{array}{c}\rho_{s} \\
\left(\mathrm{~kg} / \mathrm{m}^{3}\right)\end{array}$ & $\begin{array}{c}E_{s} \\
(\mathrm{GPa})\end{array}$ & $\begin{array}{c}\beta^{s} \\
(\mathrm{rad} / \mathrm{s})\end{array}$ & $\begin{array}{c}\beta^{p} \\
(\mathrm{rad} / \mathrm{s})\end{array}$ \\
\hline 4475 & 2700 & 68 & $5.9 \times 10^{-5}$ & $9.8 \times 10^{-5}$ \\
\hline
\end{tabular}

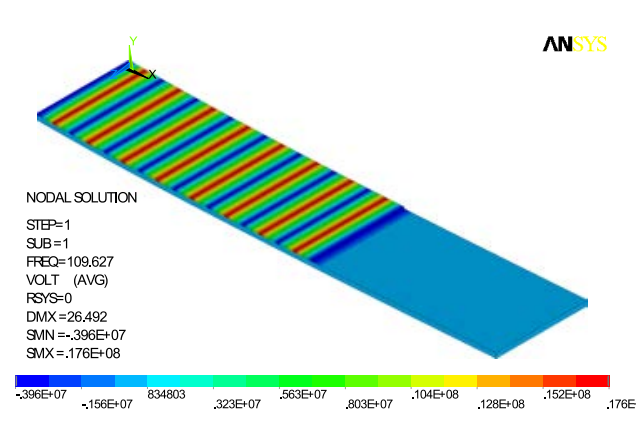

(a) Electric potential distribution

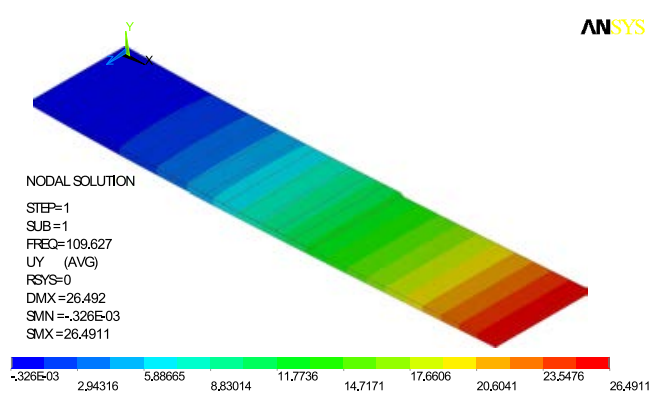

(b) Mechanical displacement along the $y$-axis

Figure 8: FEM model excited near the fundamental mode shape.

\subsection{FE Model of the PVEH}

For validation purposes, a FEM model of the PVEH is developed using the commercial package ANSYS. The model considers a homogenized material and transverse IDE as shown in Figure 6. A 3D-coupled field SOLID5 elements are used for the piezoelectric material along with circuit elements CIRCU94 to connect the external electrical circuit to the nodes representing the electrodes. The extra-shape functions option is activated. A nil potential is applied to the nodes corresponding to the negative electrodes. Also, equipotentiality condition is used for positive and negative electrodes. Material dependent damping is used in ANSYS and the corresponding material-dependent stiffness multipliers are given in Table 3. The FEM model has 22800 elements and 26313 nodes. The distribution of the electrical potential and the transverse mechanical displacement are shown in Figures 8-a and 8-b, respectively, when OC condition is considered and $N=17$ electrodes.

\subsection{Self-field effect and quadratic electric potential distribution}

A comparison between different electric field assumptions is considered in this section. In fact, if the self-field is taking into account, a quadratic electric potential distribution is obtained. If not, a linear electric potential distribution prevail [16, 25]. We calculate the electric potential obtained by the proposed analytical models and compare it with the 3D-FEM solution.

To do so, the electric potential distribution $\varphi(x, y, t)$ between transverse IDE is obtained by integrating, over the $x$ direction, the electric field $E_{1}{ }^{*}(x, y)$ given by 


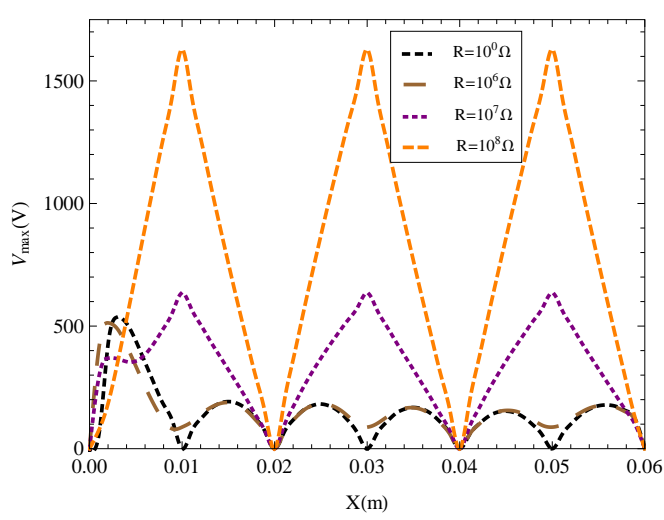

(a) Variation in the $x$-direction obtained by FEM for $y=y_{1}+\frac{h_{p}}{2}$

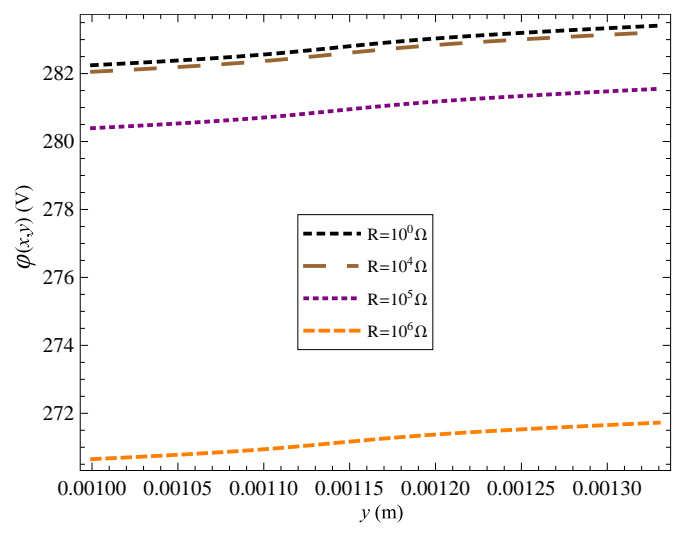

(c) Variation in the $y$-direction obtained by FEM for $x=0.025 \mathrm{~m}$

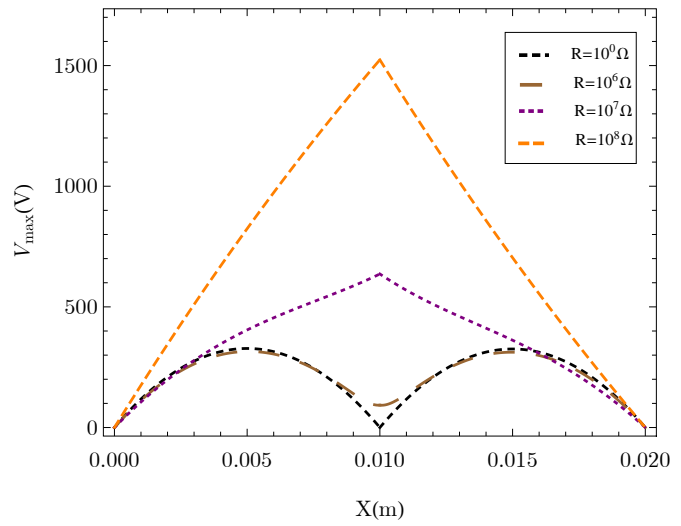

(b) Variation in the $x$-direction obtained analytically for $y=y_{1}+\frac{h_{p}}{2}$

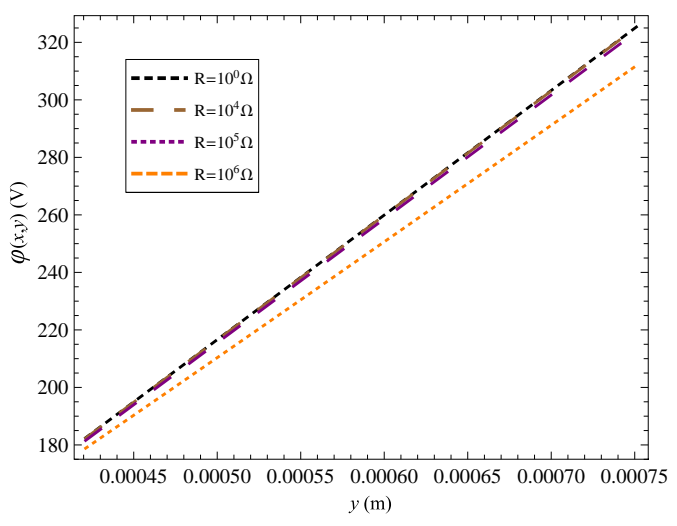

(d) Variation in the $y$-direction obtained analytically for $x=0.025 \mathrm{~m}$

Figure 9: Variation of the electrical potential in the longitudinal and transversal directions for different values of load resistance at $\omega_{S C}$, when $N=7$. 
Equation (7). That is,

$$
\begin{aligned}
\varphi(x, y, t)= & \frac{V(t)}{d}(x-i d)-\frac{\bar{e}_{33}^{e}{ }^{2}}{\bar{\epsilon}_{33}^{e}} y\left[\phi_{r}^{I^{\prime}}(x)-\phi_{r}^{I^{\prime}}(i d)\right] \eta_{r}(t) \\
& +\frac{\bar{e}_{33}^{e}{ }^{2}}{d \bar{\epsilon}_{33}^{e}} y(x-i d)\left[\phi_{r}^{I^{\prime}}((i+1) d)-\phi_{r}^{I^{\prime}}(i d)\right] \eta_{r}(t), i=0, \ldots, N-1(32
\end{aligned}
$$

Figure 9-a shows the variation of the electric potential in the $x$-direction obtained by FEM when the $y$ coordinate is fixed to $y=y_{1}+\frac{h_{p}}{2}$. Similarly, in Figure 9-b, the variation of the electric potential using Equation (32) is plotted. It is clear from both figures that the electric potential distribution follows a linear behavior only for large resistance load approaching $\mathrm{OC}$ condition $\left(\geq 10^{7} \Omega\right)$. Only in this case one can confirm that the linear assumption is valid. For resistance loads lower than $10^{7} \Omega$ the electric potential distribution is nonlinear. In this case quadratic distribution could be an adequate choice to describe potential variation between two consecutive electrodes. It is worth noting here that in Figure 9-a, the first two electrodes depict a different behavior because local effects are dominant at the clamped side of the beam. Also, it is observed that, except the first two electrodes, the proposed model provides a good qualitative as well as quantitative estimation of the electric potential variation when compared to FEM 3D model results.

On the other hand, for the $y$-direction, linear electric potential distribution is observed for all resistance loads in Figures 9-c and 9-d where FEM and analytical expression in Equation (32) have been used, respectively. For the $y$-direction, linear or quadratic assumptions could be used and both represent a correct choice. However, the validation is only qualitative for the analytical results obtained along the $y$-direction.

\subsection{Forced dynamic response of the PVEH and validation with FEM results}

We consider now the forced case where base excitation is taking into account. Figures 10 -a and 10-b show variation of the maximum peak harvested voltage $\left(V_{\max }\right)$, calculated using different models, when respectively excitation frequency $\Omega$ and resistance load $R$ are varied.

As one can see in Figure 10-a, the proposed analytical model predicts accurately the response of the PVEH for both OC and SC conditions. However, the classical analytical model with linear potential variation underestimates the harvested voltage over a broad range of electrical load resistances. Figure 10-b shows the frequency-response curves of $V_{\max }$ obtained from different models when the load resistance is $R=10^{4} \Omega$. As observed in this figure, more than $10 \%$ error is obtained for the maximum harvested voltage calculated using the classical model, while only a peak error of $3 \%$ is obtained at resonance for the proposed model.

We compare the fundamental resonance frequencies of the PVEH obtained from the analytical approach with those obtained using the FEM model. In table 4, the fundamental resonance frequencies for OC $\left(\omega_{O C} / 2 \pi\right)$ and SC $\left(\omega_{S C} / 2 \pi\right)$ conditions are shown. Also resonance frequencies obtained using a uniform cross-section mode shapes and a linear electric potential distribution, calculated by Jemai et al. [25] are included 

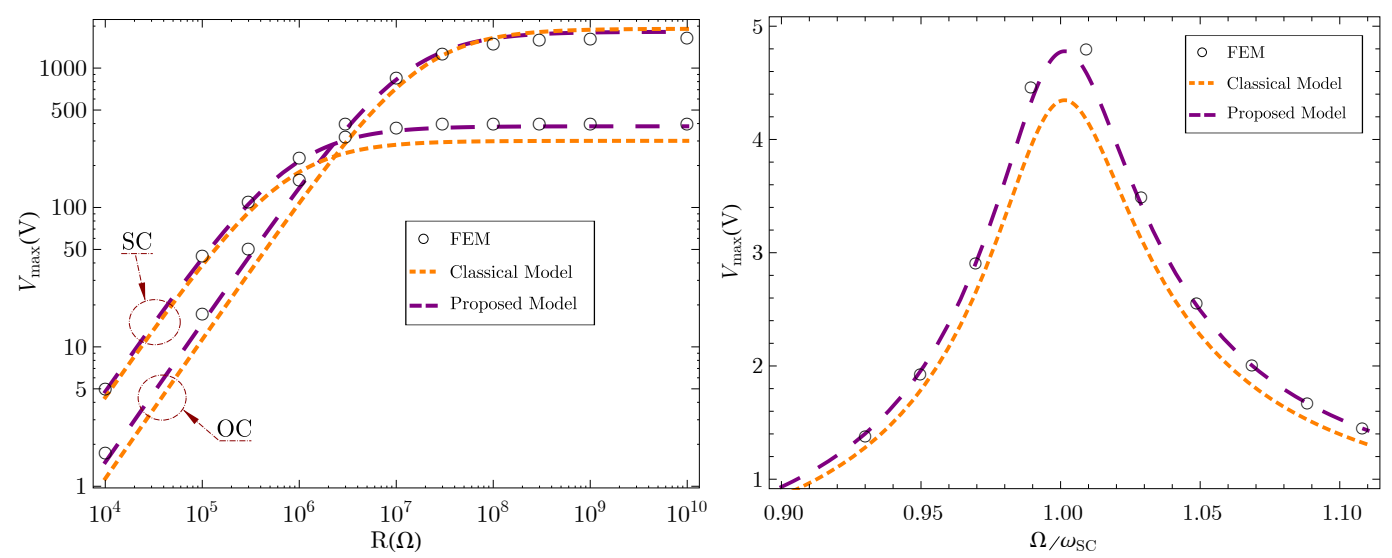

(a) Variation of $V_{\max }$ versus load resistance $R$ for (b) Frequency-response curves of $V_{\max }$ at $R=$ OC and SC conditions $10^{4} \Omega$.

Figure 10: Dynamic response validation of the energy harvester with FEM results, when $\mathrm{N}=31$.

in the same table. FEM has been taking as a reference model to compare different approaches. The displayed results show that the proposed analytical model, corresponding to non-uniform cross-section mode shapes and quadratic electric potential assumption, provides results in good agreement with FEM with approximately $1 \%$ error for both OC and SC conditions. On the other hand, between $7 \%$ and $11 \%$ error is obtained when linear electric field and uniform cross-section mode shape assumptions are considered.

Table 4: OC and SC resonance frequencies: comparisons between different models, when $\mathrm{N}=31$.

\begin{tabular}{|c|c|c|c|c|}
\hline & $\omega_{S C} / 2 \pi(\mathrm{Hz})$ & Error \% & $\omega_{O C} / 2 \pi(\mathrm{Hz})$ & Error \% \\
\hline Classical model [25] & 89.21 & 11.73 & 101.41 & 7.49 \\
\hline Proposed model & 100.09 & 0.85 & 110.51 & 1.19 \\
\hline FEM & 100.95 & - & 109.2 & - \\
\hline
\end{tabular}

\section{Parametric and performance analysis of the AFC-based PVEH}

In this section only the analytical model corresponding to the quadratic electrical potential assumption and nonuniform cross-section mode shapes will be considered to perform a parametric design analysis of the PVEH. The analysis will cover the influence of the number of electrodes, the thickness ratio, the length ratio, the substrate material and the FVF. 


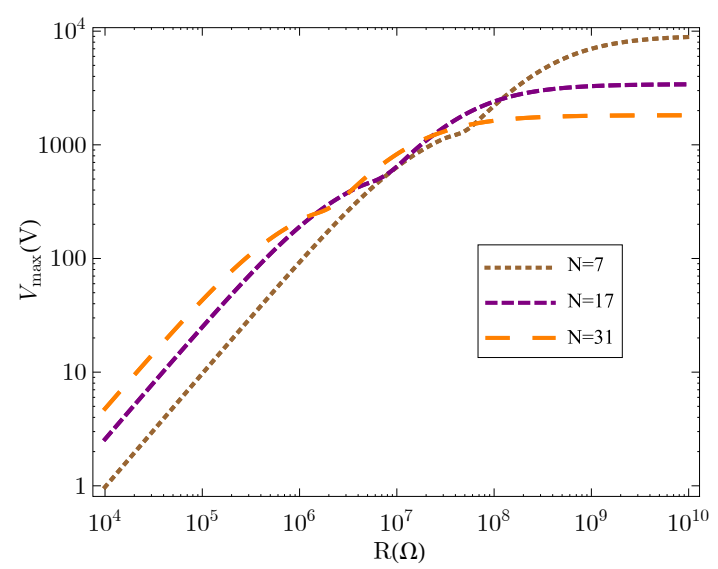

(a) Harvested voltage.

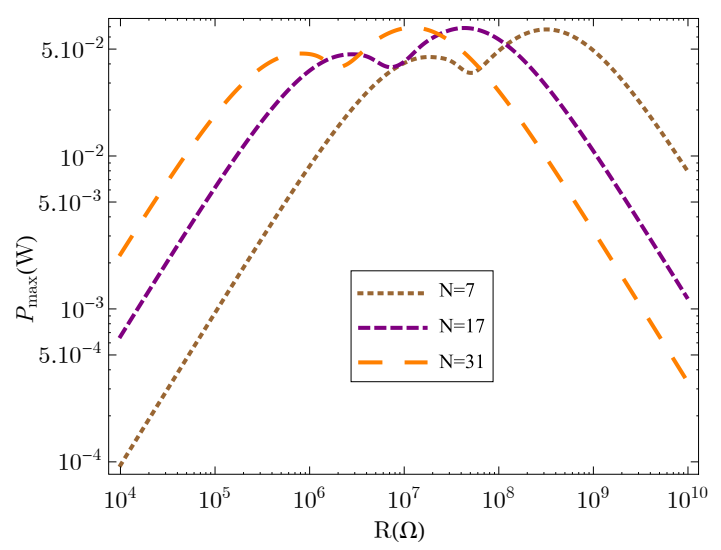

(c) Tip amplitude.

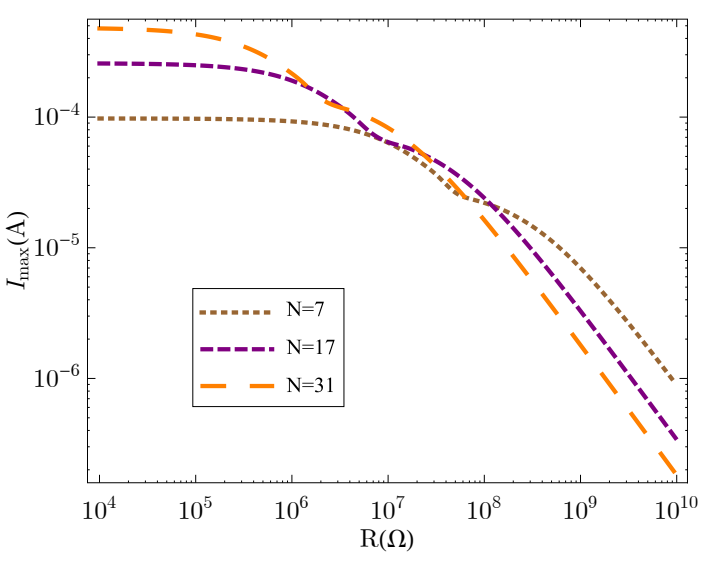

(b) Harvested electrical current.

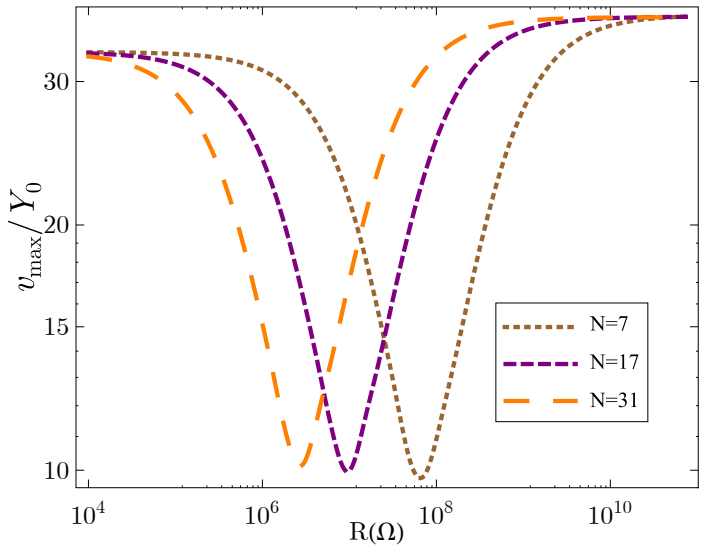

(d) Harvested power.

Figure 11: Analytical and numerical results for $N=7, N=17$ and $N=31$ IDE.

\subsection{Influence of the number of electrodes}

In Figures 11 the results obtained from the proposed analytical solutions are displayed for the harvested voltage $V_{\max }$, electrical current $I_{\max }$, tip amplitude $v_{\max }$ and harvested power $P_{\max }$. The $\max$ subscript indicates that the corresponding value has been calculated as the maximum peak for a frequency sweep from $\omega_{S C}$ to $\omega_{O C}$. In these figures the electric load resistance $R$ is varied for different numbers $N$ of IDE.

If large electrical resistance loads are considered (larger than $10^{8} \Omega$ ), the harvested voltage decreases when the number of electrodes increases (Figure 11-a). The same behavior can be observed for the electrical current as shown in Figure 11-b. As a result, a larger maximum electrical harvested power is obtained for the smallest number of electrodes. The OC voltages are $9155.78 \mathrm{~V}, 3417.94 \mathrm{~V}$ and $1820.85 \mathrm{~V}$ for $N=7, N=17$ and $N=31$, respectively. On the other side, for low values of load resistance, increasing the number of electrodes $N$ results in an increase of the electrical current and voltage as shown in Figures 11-a and 11-b.

It can be observed from Figures 11-a-b that for load resistance conditions close to 
OC, we found that the voltage is inversely proportional to the number of electrodes $N$. According to Ohm's law across the resistor the same behavior is observed for the electrical current as the number of electrodes is varied. In fact, for large resistance loads, the electric displacement is negligible i.e. $D_{1} \approx 0$. Therefore, if one neglects the self-field effect in Equation (7), the electric field can be approximated by $E_{1}=$ - $(N-1) \frac{V(t)}{L_{1}}$. Similarly, the normal stress can be approximated by an equivalent normal force $F_{N}(t)$, therefore $F_{N}(t)=\sigma_{11}^{p} b h_{p}$. From Equations (3) and (4) the harvested voltage as a function of the stress can then be deduced as follows

$$
V(t)=\frac{L_{1} F_{N}(t) K_{33}^{2}}{(N-1) b h_{p} \bar{e}_{33}^{e}}
$$

where $K_{33}^{2}=\frac{1}{1+\left(\frac{\bar{C}_{33}^{e} \epsilon_{33}^{e}}{\bar{e}_{33}^{e}{ }^{2}}\right)}[31]$. On the other side of Figures 11-a-b where load re-

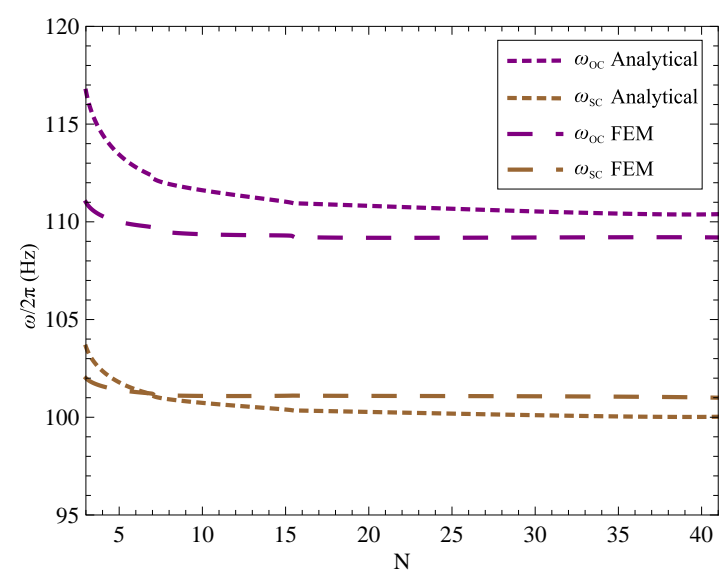

Figure 12: Variation of the $\mathrm{OC}$ and SC fundamental resonance frequencies with the number of IDE $N$.

sistance is close to SC conditions, we demonstrate that the electrical current is proportional to the number of electrodes and according to the Ohm's law the voltage will behave the same way. In fact, for SC conditions, the electrical field is negligible $E_{1} \approx 0$. Since the electrical current can be defined by $I(t)=\sum_{i=1}^{N-1} \int_{A} \frac{d}{d t} D_{1} d A$ and using Equations (3) and (4) for SC conditions, we end up with the following approximation of the generated electrical current:

$$
I(t)=\frac{\bar{e}_{33}^{e}}{\bar{C}_{33}^{e}} \sum_{i=1}^{N-1} \int_{A} \frac{d \sigma_{11}^{p}}{d t}(x, t) d A=(N-1) \frac{\bar{e}_{33}^{e}}{\bar{C}_{33}^{e}} \frac{d F_{N}(t)}{d t}
$$

The harvested power for different number of electrodes is shown in Figure 11-c where the the electric load resistance $R$ is varied for different numbers of IDE. The maximum harvested power is obtained for the optimal resistance load. This latter is bigger as the number of IDE is reduced. 
The results mentioned above are in agreement with those obtained by Sodano et al. [16] where the authors found that the reduced effective capacitance is the principal drawback of IDE configuration. This observation was proven by the fact that in general IDE configurations depict large number of electrodes, and thus large impedance is obtained due to small effective capacitance and small excitation frequencies [16]. As long as the maximum power generation of the PVEH is obtained when impedance matching is ensured, a lower number of electrodes could have improved the performance of their PVEH.

Concerning the tip amplitude of the beam shown in Figure 11-d, we note that the amplitude of motion can increase or decrease with $N$ whether the load resistance $R$ is high or low, respectively. However, for resistance loads close to OC and SC conditions, the tip amplitude is not sensitive to variation of the number of electrodes $N$.

The influence of the number of electrodes $N$ on the $\mathrm{OC}$ and SC fundamental resonance frequencies $\left(\omega_{O C}\right.$ and $\left.\omega_{S C}\right)$ of the PVEH are studied in Figure 12. Both $\omega_{O C}$ and $\omega_{S C}$ decrease with the number of electrode. The variation almost stabilize past $N=25$ IDE.

\subsection{Influence of the thickness ratio $\lambda_{h}$}

We analyze in this section the influence of the thickness ratio $\lambda_{h}=\frac{h_{p}}{h}$ on the performance of the PVEH. The choice of a correct substrate thickness is necessary to let the active layer benefit from high strains far from the neutral axis. In order to vary $\lambda_{h}$, we fix the value of the thickness $h=1.33 \mathrm{~mm}$ and vary the thickness of the AFC patch.

Figures 13 represent the analytical variation of $P_{\max }$ and $v_{\max }$ at $\omega_{S C}$, near the first mode, as $\lambda_{h}$ is varied for different load resistances. For $R \leq 10^{7} \Omega$, the electrical harvested power is characterized by a unique maximum, beyond the variation becomes monotonic (Figure 13-a). When $\lambda_{h}$ goes to zero the AFC layer becomes very small, as a result the harvested power is substantially reduced. When $R$ increases, the peak is progressively switched to lower values of $\lambda_{h}$ until its disappearance when the variation becomes monotonic for $R \geq 10^{8} \Omega$. When $\lambda_{h}$ gets larger and close to 1 , the neutral axis moves into the AFC layer, thus charge cancellation occurs and the harvested power goes to zero.

Alike the harvested power case, the tip amplitude variation as function of $\lambda_{h}$ is shown in Figure 13-b. For high values of load resistance $\left(R \geq 10^{7} \Omega\right)$, the tip amplitude presents a minimum value around $\lambda_{h}=0.4$. This minimum tends to disappear and the variation becomes monotonic as the load resistance $R \leq 10^{5} \Omega$.

\subsection{Influence of the length ratio $\delta_{h}$}

Next, we look at the influence of the length ratio $\delta_{L}=\frac{L_{1}}{L}$ on the performances of the PVEH. The optimal length of the AFC patch is studied here using a parametric analysis. We vary the length of AFC patch $L_{1}$ and keep $L$ equal to $100 \mathrm{~mm}$. Figures 14 represents the analytical variation of the maximum harvested power at $\omega_{S C}$ near the first mode as $\delta_{L}$ is varied for different load resistances.

The curves, in Figure 14-a, demonstrate that the harvested power variation with respect of the length ratio observe an optimal value around $\delta_{L}=0.8$ as long as the load 


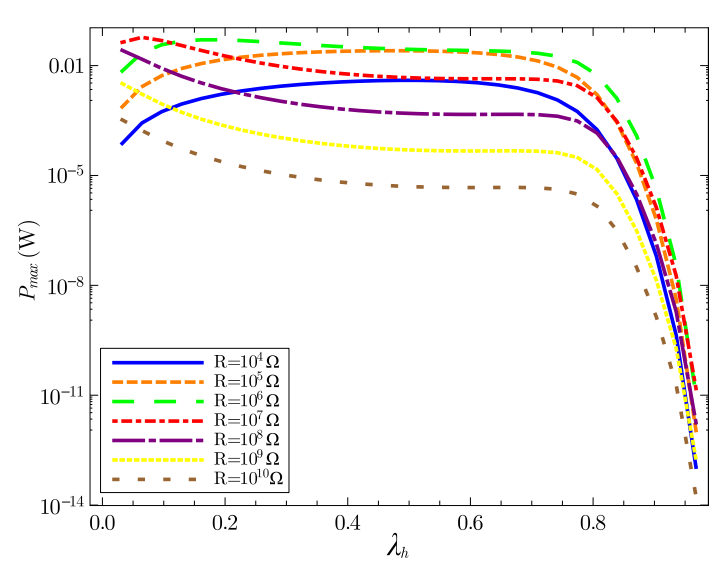

(a) Harvested Power.

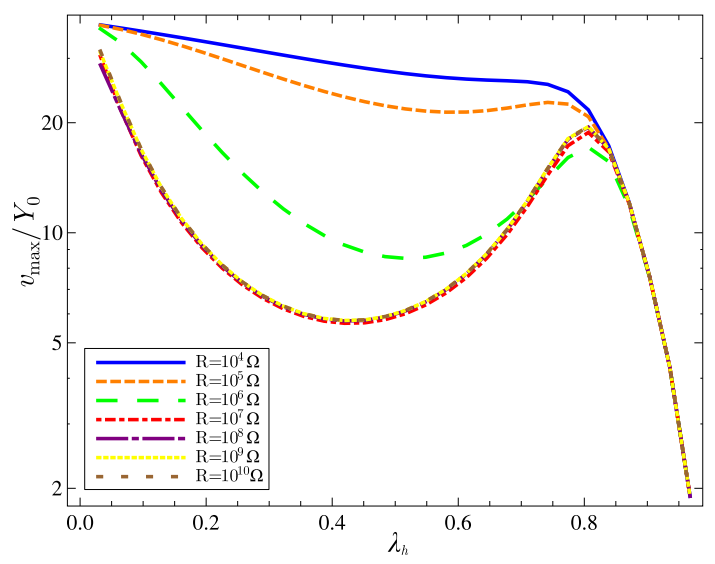

(b) Tip displacement.

Figure 13: Variation of analytical harvested power and tip amplitude with thickness ratio $\lambda_{h}$ at $\omega_{S C}$ for different load resistances, when $N=31$ IDE.

resistance $R \geq 10^{7} \Omega$. For lower values of $R$, the optimal value of $\delta_{L}$ decreases as the load resistance is decreasing. Also, due to the change of value of the optimum length, intersections between curves appear, giving the same harvested power for different values of load resistance.

Concerning the tip deflection of the beam $v_{\max }$, shown in Figure 14-b, we can note that for high values of load resistance $\left(R \geq 10^{7} \Omega\right)$, the tip deflection is insensitive to load resistance variation. The behavior is different, when the load resistance is $R<10^{7} \Omega$.

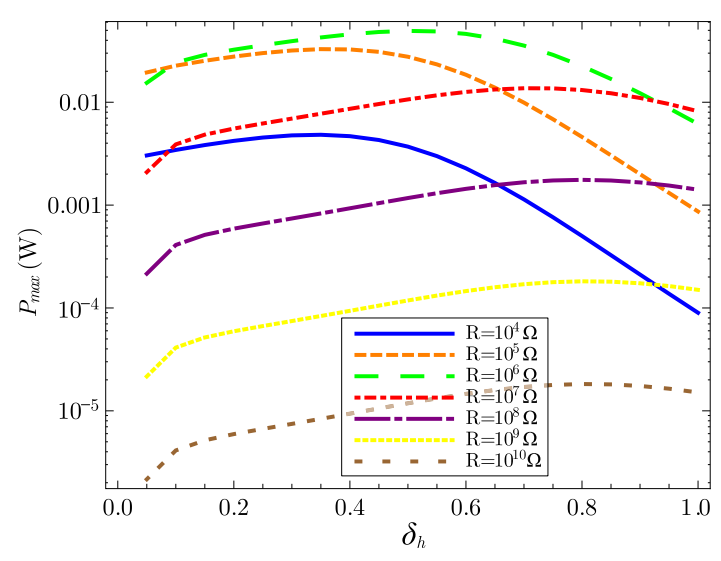

(a) Harvested Power.

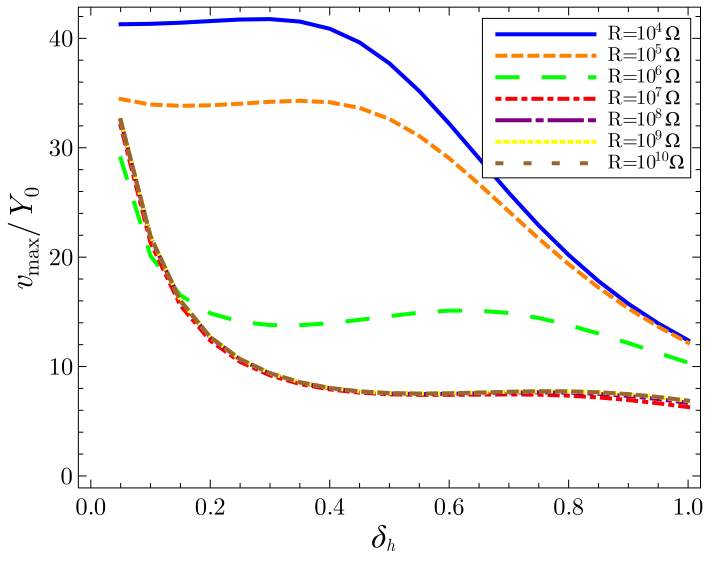

(b) Tip displacement.

Figure 14: Variation of analytical harvested power and tip amplitude with length ratio $\delta_{h}$ at $\omega_{S C}$ for different load resistances, when $N=31$ IDE. 


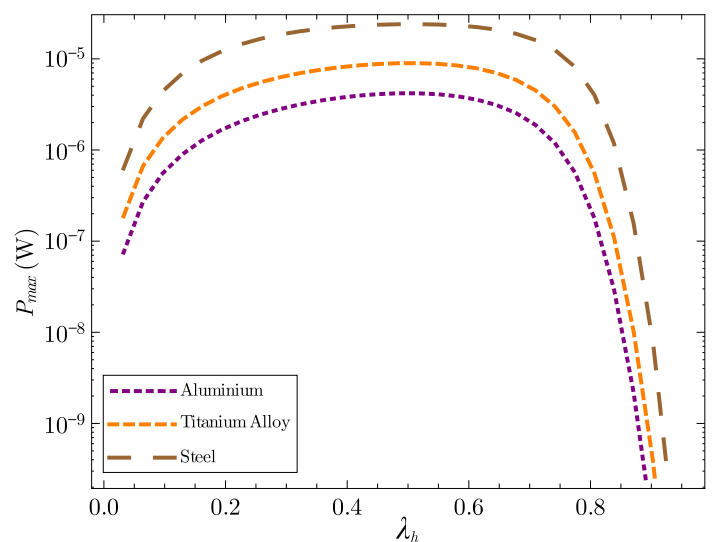

(a) $R=10^{1} \Omega$.

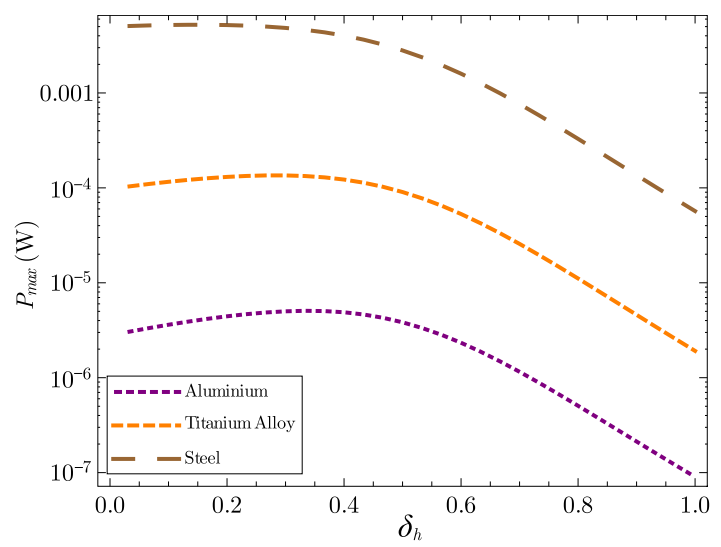

(c) $R=10^{1} \Omega$.

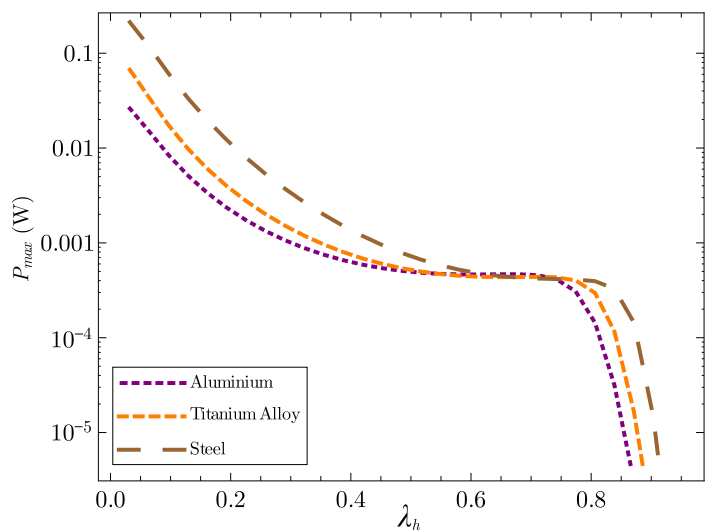

(b) $R=10^{8} \Omega$.

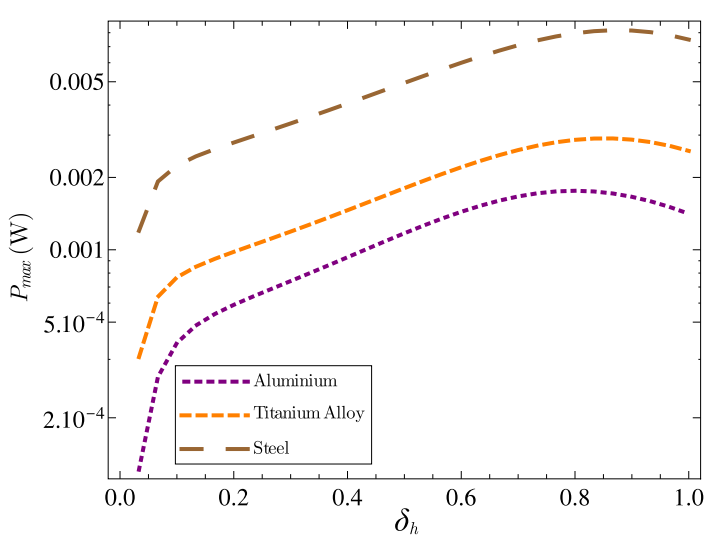

(d) $R=10^{8} \Omega$.

Figure 15: Analytical harvested power at $\omega_{S C}$ for different substrate materials, $N=31$ IDE.

\subsection{Influence of the substrate material}

The choice of the substrate material can have not only an influence on the effective stiffness and the natural frequencies of the PVEH but can also act upon its overall performance. Figures 15 shows the analytical variation of the harvested power as we vary the thickness ratio $\lambda_{h}$ and the length ratio $\delta_{h}$ for different substrate materials when $R=10^{1} \Omega$ in Figures 15 -a-b and $R=10^{8} \Omega$ in Figures 15 -c-d. We give in Table 5 the properties of different materials used for the substrate layer in this section.

When the substrate layer gets stiffer, the neutral axis moves down increasing the strain inside the AFC patch and therefore the harvested power increases. This behavior is observed for both SC and OC conditions in Figures 15. However, for OC condition $\left(R=10^{8} \Omega\right)$ and medium values of the thickness ratio (between 0.6 and 0.8 ), this behavior is not valid and the opposite effect is observed. Indeed, as a softer and lighter material is used, the piezoelectric layer dominates the beam stiffness, thereby generating more harvested power. 
Table 5: Material properties for substrate layer

\begin{tabular}{|c|c|c|c|}
\hline & Aluminum & Titanium Alloy & Steel \\
\hline The elastic modulus $(\mathrm{GPa})$ & 68 & 100 & 207 \\
\hline The mass density $\left(\mathrm{kg} / \mathrm{m}^{3}\right)$ & 2700 & 4650 & 7850 \\
\hline The Poisson's ratio & 0.3 & 0.36 & 0.27 \\
\hline
\end{tabular}

\subsection{Influence of the fiber volume fraction}

The increase of the piezoelectric material inside the AFC patch is traditionally linked to an increase of the harvested energy. To verify this assumption, we plot in Figures 16 the analytical variation of the harvested power as we vary the thickness ratio $\lambda_{h}$ and the length ratio $\delta_{h}$ at $\omega_{S C}$ for different fiber volume fraction (FVF) when $R=10^{1} \Omega$ in Figures 16-a-c and $R=10^{8} \Omega$ in Figures 16-b-d, using 31 IDE.

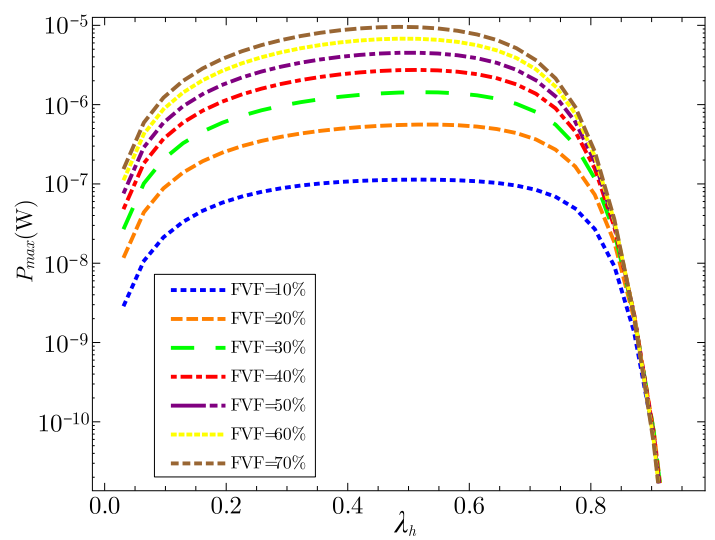

(a) $R=10^{1} \Omega$.

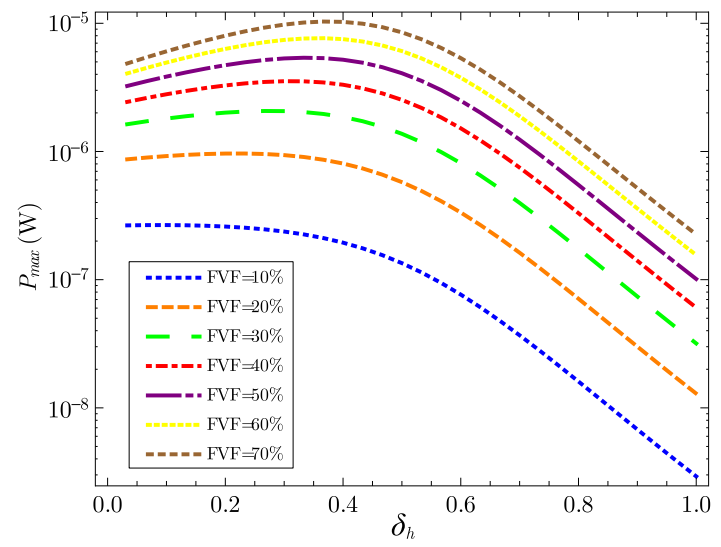

(c) $R=10^{1} \Omega$.

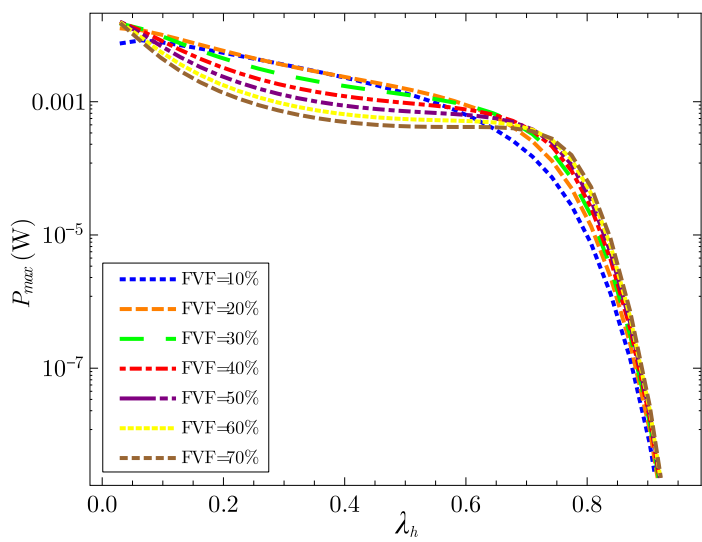

(b) $R=10^{8} \Omega$.

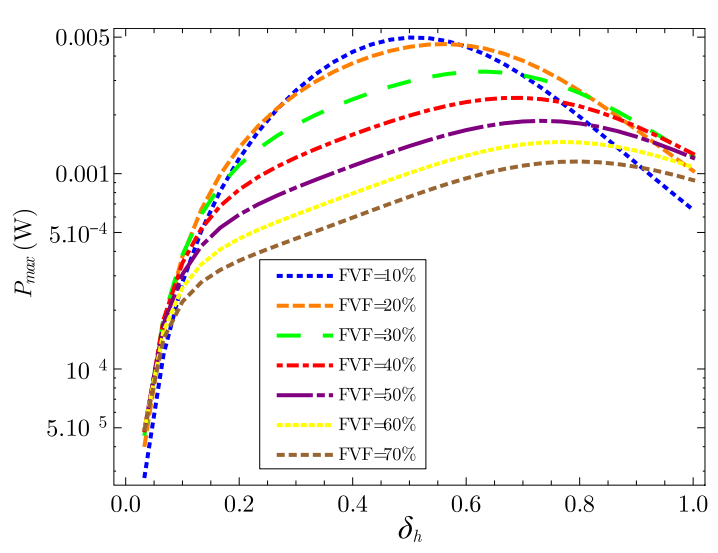

(d) $R=10^{8} \Omega$.

Figure 16: Analytical harvested power at $\omega_{S C}$ for different $\mathrm{FVF}, N=31 \mathrm{IDE}$.

For low values of load resistance $\left(R=10^{1} \Omega\right)$, as expected, the harvested power increases with the increase of the FVF. However, for high values of load resistance 
$\left(R=10^{8} \Omega\right.$ ), the variation is of the harvested power is reversed around the values of the thickness ratio and the length ratio in the intervals $0.1 \leq \lambda_{h} \leq 0.6$ and $0.3 \leq \delta_{h} \leq 0.6$. In this range, an increase of the FVF conducts to a decrease of the harvested energy. In fact, as the FVF gets larger, the stiffness of the AFC layer grows, thus the neutral axis moves up into the AFC layer and the resulting charge cancellation reduces the harvested energy.

\section{Comparative analysis of different PVEH designs}

The analysis presented in this section compares several PVEH designs, with IDE $\left(d_{33}\right.$ mode) and with standard electrodes ( $d_{31}$ mode). Three different piezoelectric devices are chosen in order to determine the impact of the design on the generated power by the PVEH. The chosen designs are the AFC with 50\% PZT ( $N=31$ IDEs) denoted here as "50-IDE", a 100\% monolithic PZT PVEH with IDE ( $N=31$ IDEs) denoted as "100-IDE", this design is similar to the Quick Pack IDE used by Sodano et al. [19] and the third design uses standard electrodes with $100 \%$ monolithic PZT and $d_{31}$ mode, denoted by "100-STD", equivalent to the standard Quick Pack also used by used in [19]. The dimensions of the proposed devices are shown in Table 5.

Table 6: Material and geometrical properties of the three PVEH: 50-IDE, 100-IDE and 100-STD.

\begin{tabular}{|c|c|c|c|c|c|c|c|}
\hline $\begin{array}{c}L \\
(\mathrm{~mm})\end{array}$ & $\begin{array}{c}L_{1} \\
(\mathrm{~mm})\end{array}$ & $\begin{array}{c}b \\
(\mathrm{~mm})\end{array}$ & $\begin{array}{c}h_{p} \\
(\mathrm{~mm})\end{array}$ & $\begin{array}{c}h_{s} \\
(\mathrm{~mm})\end{array}$ & $\begin{array}{c}E_{s} \\
(\mathrm{GPa})\end{array}$ & $\begin{array}{c}\rho_{s} \\
\left(\mathrm{~kg} / \mathrm{m}^{3}\right)\end{array}$ & $\zeta_{1}$ \\
\hline 100 & 100 & 20 & 0.4 & 0.5 & 100 & 7165 & 0.01 \\
\hline
\end{tabular}

Performances of the three proposed devices are shown in Figures 17 where harvested voltage, electrical current and the harvested power (in normalized forms) are plotted versus the load resistance $R$. Simulations of the 50-IDE and 100-IDE devices are carried out using our proposed analytical and numerical models. Whereas, the results corresponding to the 100-STD device are obtained using the model developed by Erturk et al [32] and our 3D FE model. Very good agreement are obtained between numerical and analytical results.

In Figure 17-a one can remark that the OC voltage delivered by the 50-IDE and the 100-IDE devices are respectively about 21 and 19 times higher than the one obtained for the 100-STD. The results are consistent with reported studies that showed approximately 20 times greater voltage for IDE [21, 12]. Similarly, the results shown in Figure 17-b demonstrate that the harvested current for the 50-IDE and the 100-IDE devices are respectively about 10 and 5 times lower than the 100-STD device. These results are in line with those obtained by $[19,21]$.

As demonstrated in Figures 17, the electrical output from devices using IDE produce very large voltage but an extremely low current, compared to devices with standard electrode configuration. Nevertheless, it may be observed that the maximum optimum power is approximatively the same when similar amount of active material is used (100-IDE and 100-STD). Song et al. [15] obtained the same conclusions when 


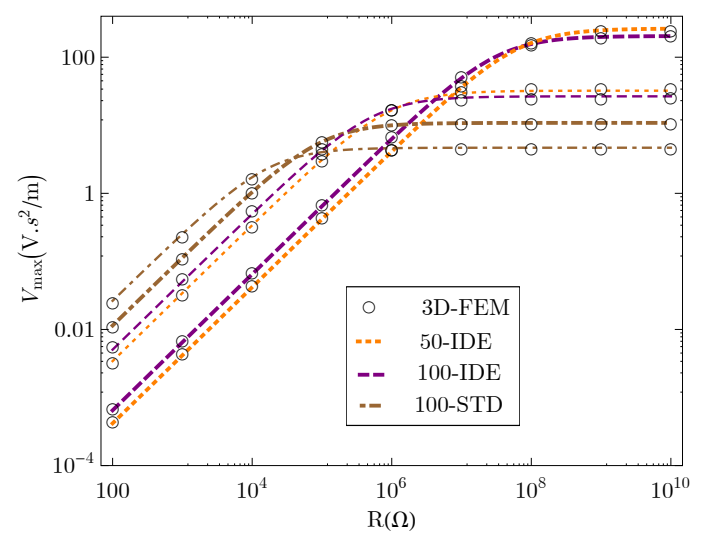

(a) Harvested voltage.

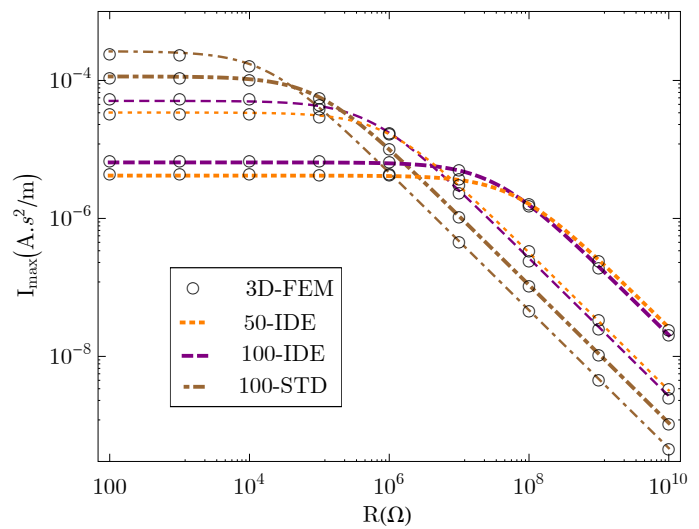

(b) Harvested electrical current.

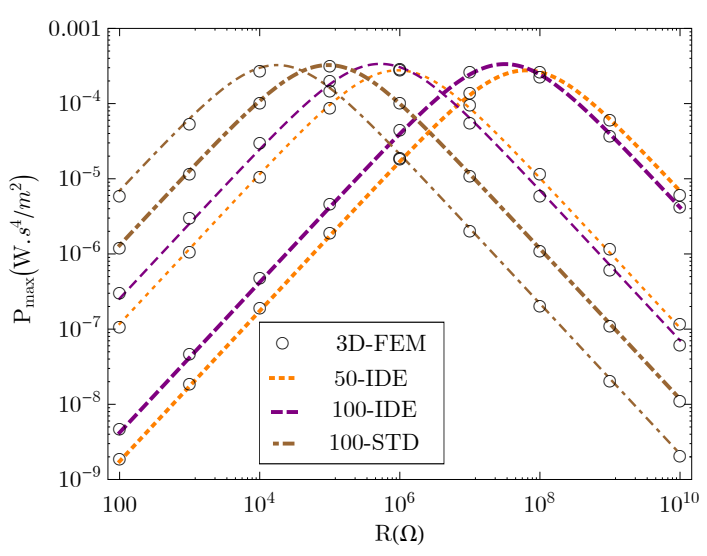

(c) Harvested power.

Figure 17: Analytical and numerical results of the three PVEH designs. Thin curves denote SC conditions and thick curves denote OC conditions. 
comparing these devices. However, it is worth noting here that conducting experimental analysis, Sodano et al. [16] found that the maximum power outputs of the MFC with IDE and $82 \% \mathrm{FVF}$ and the Quick pack IDE were small relative to the standard Quick pack with classical electrodes and 100\% PZT (17 times lower). On the other hand, comparing $d_{31}$ and $d-33$ modes in PVEH, Kim et al. [33] found that comparable harvested power are observed experimentally when the geometry of the electrodes are correctly tuned. The findings of Kim et al. [33] confirms our results and proves that an optimization of the number of electrodes and their geometry can drastically increase the performance of the $d-33$-based PVEH.

\section{Conclusion}

We have developed a parameterized analytical model of a vibration-based energy harvester using piezocomposite material and interdigitated electrode. The piezocomposite is taken to be similar to the Active Fiber Composite (AFC), a commercially available piezocomposite actuator. Since the AFC patch is homogenized using an FEM based technique and replaced by an equivalent piezoelectric material, other piezocomposite designs can be used in the proposed modeling process, such as the Micro Fiber Composite (MFC). The interdigitated electrode configuration have been maintained in the proposed model. Also, the model takes into account the fact that the electric potential field variation between two consecutive electrodes is not uniform. It has been modeled using a quadratic variation that takes into account the self-field effect of the piezoelectric material.

The proposed energy harvester has a unimorph design with a metallic substrate layer partially covered by an AFC patch. A Galerkin procedure has been applied to discretize the partial differential equations describing the mechanical and electrical behaviors of the energy harvester. The test functions have been chosen to be the mode shapes of the structure including the non-uniform cross-section variation along the beam length. A closed-form solutions of the generated power, the harvested voltage, the electrical current and the tip amplitude was carried out. The results have been first validated by a $\mathrm{FE}$ analysis using $3 \mathrm{D}$ model of the energy harvester. A parametric study was performed to investigate the effects of various physical and geometrical parameters on the harvested power and displacement. It was shown that the developed analytical model can be used to improve the energy harvesting performance of the interdigitated piezoelectric system by selecting the correct design parameters such as the number of electrodes, the thickness ratio, the length ratio, the fiber volume fraction and the substrate material. And that correctly chose these parameters can drastically increase or decrease the harvested electrical power.

\section{Acknowledgments}

The first three authors are grateful for the funding provided to their laboratory by the Tunisian Ministry of Higher Education and Scientific Research. This work was performed under the framework of the NSF International Institute for Multifunctional 
Materials for Energy Conversion (NSF IIMEC); all the authors would like to acknowledge the financial support by NSF, Grant No. DMR - 0844082. The authors would like also to thank Mr. Hassene Ben Atitallah from Penn State University for the SEM pictures.

\section{References}

[1] S. Jiang, X. Li, S. Guo, Y. Hu, J. Yang, Q. Jiang, Performance of a piezoelectric bimorph for scavenging vibration energy, Smart Materials and Structues 2005;14:769-74.

[2] S. Beeby, N. White, 2010, Energy harvesting for autonomous systems, Artech House.

[3] S. Roundy, E.S. Leland, J. Baker, E. Carleton, E. Reilly, E. Lai, B. Otis, J.M. Rabaey, P.K. Wright, V. Sundararajan, Improving power output for vibrationbased energy scavengers, IEEE Pervasive Computing 2005;4:28-36.

[4] S. Ben Ayed, A. Abdelkefi, F. Najar, M.R. Hajj, Design and Performance of Variable-Shaped Piezoelectric Energy Harvesters, Journal of Intelligent Material Systems and Structures 2014;25:174-186.

[5] A. Abdelkefi, F. Najar, A.H. Nayfeh, S. Ben Ayed, An energy harvester using piezoelectric cantilever beams undergoing coupled bending-torsion vibration, Smart Materials and structures 2011;20:115007(11pp).

[6] A. Abdelkefi, A.H. Nayfeh, M.R. Hajj, F. Najar, Energy Harvesting from a Multifrequency Response of a Tuned Bending-torsion System, Smart Materials and Structures 2015;21:075029(9pp).

[7] Q. Zheng, Y. Xu, Asymmetric air-spaced cantilevers for vibration energy harvesting, Smart Materials and structures 2008;17:6.

[8] A. Erturk, D.J. Inman, An experimentally validated bimorph cantilever model for piezoelectric energy harvesting from base excitations, Smart Materials and structures 2009;18:18.

[9] A. Erturk, P.A. Tarazaga, J.R. Farmer, D.J. Inman, Effect of Strain Nodes and Electrode Configuration on Piezoelectric Energy Harvesting From Cantilevered Beams, Journal of Vibration and Acoustics 2009;131.

[10] C. R. Bowen, A. Bowles, S. Drake, N. Johnson, S. Mahon, Fabrication and finite element modelling of interdigitated electrodes, Ferroelectrics 1999;228:257-269.

[11] R. Sood, T.B. Jeon, J.H. Jeong, S.G. Kim, Piezoelectric micro power generator for energy harvesting, Proceedings Technical Digest of the 2004 Solid-State Sensor and Actuator Workshop. 
[12] Y.B. Jeon, R. Sood, H.H. Jeong, S.G Kim, MEMS power generator with transverse mode thin film PZT, Sensors Actuators A 2005;122:16-22.

[13] A.A. Bent, N.W. Hagood, J.P. Rodgers, Anisotropic actuation with piezoelectric fiber composites, Journal of Intelligent Material Systems and Structures 1995;6:338-349.

[14] W.K. Wilkie, R.G. Bryant, J.W. High, R.L. Fox, R.F. Hellbaum, A. Jalink, B.D. Little, P.H. Mirick, Low-Cost Piezocomposite Actuat or for Structural Control Applications, Proceedings of 7th SPIE International Symposium on Smart Structures and Materials, Newport Beach, CA, March 5-9, 2000.

[15] J.H. Song, Y-.T Choi, N.M. Wereley, A.S. Purekar, Energy Harvesting Devices Using Macro-fiber Composite Materials, Journal of Intelligent Material Systems and Structures 2005;21:647-658.

[16] H.A. Sodano, , J.M. Lloyd, , D.J. Inman, An Experimental Comparison Between Several Active Composite Actuators For Power Generation, Smart Materials and Structures 2006;15:1211-1216.

[17] H.A. Sodano, G. Park, D.J. Inman, Estimation of electric charge output for piezoelectric energy harvesting, Journal of Strain 2004;40:49-58.

[18] H.A. Sodano, G. Park, D.J. Leo, D.J. Inman, Use Of Piezoelectric Energy Harvesting Devices For Charging Batteries, SPIE's 10th Annual International Symposium on Smart Structures and Materials 2003;52:8-11.

[19] H.A. Sodano, D.J. Inman and G. Park, Comparison of Piezoelectric Energy Harvesting Devices for Recharging Batteries, Journal of Intelligent Material Systems and Structures 2005;6:799-807.

[20] W. Beckert, W.S. Kreher, Modelling piezoelectric modules with interdigitated electrode structures, Computational Materials Science 2003;26:36-45.

[21] C. Mo, S. Kim, W. Clark, Theoretical analysis of energy harvesting performance for unimorph piezoelectric benders with interdigitated electrodes, Smart Materials and Structures 2009;18:055017(8pp).

[22] C. Mo, S. Kim, W. Clark, Analysis of Power Generating Performance for Unimorph Cantilever Piezoelectric Beams with the Interdigitated Electrode, in Proc. of IDETC/CIE, ASME, (2005) San Diego, USA.

[23] R.R. Knight, C. Mo, W. Clark, MEMS interdigitated electrode pattern optimization for a unimorph piezoelectric beam, Journal of Electroceramics 2011;26:14-22.

[24] A. Erturk, O. Bilgen, M. Fontenille, D.J. Inman, Piezoelectric Energy Harvesting from Macro-Fiber Composites with an Application to Morphing-Wing Aircrafts, 19th International Conference on Adaptive Structures and Technologies, (2008) October 6-9, Ascona, Switzerland. 
[25] A. Jemai, F. Najar, M. Chafra, Z. Ounaies, Mathematical Modeling of an ActiveFiber Composite Energy Harvester with Interdigitated Electrodes, Journal of Shock and Vibration, Article ID 971597 2014;:9pp.

[26] A. Jemai, F. Najar, M. Chafra, Z. Ounaies, Modeling and nonlinear dynamics of an Active-Fiber Composite energy harvester with Interdigitated Electrodes. In the IEEE International Conference on Composite Materials \& Renewable Energy Applications (ICCMREA), (2014) January 22-24, Sousse, Tunisia.

[27] R. Masana, M.F. Daqaq, Modeling and non-linear analysis of axially loaded energy harvesters, Journal of Vibration and Acoustics 2011;133:1-10.

[28] A.B. Alamin Dow, M. Schneider, D. Koo, H.A. Al-Rubaye, A. Bittner, U. Schmid, N. Kherani, the performance of a micromachined piezoelectric energy harvester, Microsystem Technologies 2012;18:10351043.

[29] J.D. Hobeck, D.J. Inman, Artificial piezoelectric grass for energy harvesting from turbulence-induced vibration, Smart Materials and Structures 2012;21:105024.

[30] H. Berger, S. Kari, U. Gabbert, R. Rodriguez-Ramos, , R. Guinovart, J.A. Otero, J. Bravo-Castillero, An analytical and numerical approach for calculating effective material coefficients of piezoelectric fiber composites. International Journal of Solids and Structures 2005;42:5692-5714.

[31] M.A. Trindade, A. Benjeddou, Effective electromechanical coupling coefficients of piezoelectric adaptive structures: critical evaluation and optimization, Mechanics of Advanced Materials and Structures 2009;16:210-223.

[32] A. Erturk, D.J. Inman, A Distributed Parameter Electromechanical Model for Cantilevered Piezoelectric Energy Harvesters, Journal of Vibration and Acoustics 2008;130:041002.

[33] S. B. Kim, H. Park, S. H. Kim, H. C. Wikle, J. H. Park, D. J. Kim, Comparison of MEMS PZT Cantilevers Based on $d_{31}$ and $d_{33}$ Modes for Vibration Energy Harvesting, Journal of Microelectromechanical Systems, 2013;22(1):26-33. 